\title{
Kernos
}

Revue internationale et pluridisciplinaire de religion grecque antique

$17 \mid 2004$

Varia

\section{Die Thesmophoria, Brimo, Deo und das Anaktoron: Beobachtungen zur Vorgeschichte des Demeterkults}

Catherine Trümpy

\section{(2) OpenEdition}

\section{Journals}

Édition électronique

URL : http://journals.openedition.org/kernos/1399

DOI : 10.4000/kernos.1399

ISSN : 2034-7871

Éditeur

Centre international d'étude de la religion grecque antique

Édition imprimée

Date de publication : 1 janvier 2004

ISSN : 0776-3824

Référence électronique

Catherine Trümpy, « Die Thesmophoria, Brimo, Deo und das Anaktoron: Beobachtungen zur Vorgeschichte des Demeterkults », Kernos [En ligne], 17| 2004, mis en ligne le 22 avril 2011, consulté le 19 avril 2019. URL : http://journals.openedition.org/kernos/1399 ; DOI : 10.4000/kernos.1399 


\section{Die Thesmophoria, Brimo, Deo und das Analktoron: Beobachtungen zur Vorgeschichte des Demeterkults}

Der Kult der Demeter und ihrer Tochter Kore bzw. Persephone hat immer wieder, weit über den Kreis der Spezialisten hinaus, reges Interesse geweckt. ${ }^{1}$ Seit der Publikation der in den Jahren 1993-1995 gefundenen mykenischen Dokumente aus Theben, ${ }^{2}$ wo die Herausgeber u.a. eine Göttin $M \bar{a} G \bar{a}$ 'Mutter Erde' und deren Tochter Korwa (= Kore) zu finden glauben und diese mit Demeter und Kore gleichsetzen, ${ }^{3}$ ist die prähistorische Dimension des Demeterkults vermehrt ins Blickfeld gerückt und verdient zweifelsohne eine besonders sorgfältige Prüfung angesichts der neueren Forschung, die auch anderweitig zahlreiche neue Erkenntnisse über die Vor- und Frühgeschichte der Griechen vorzuweisen hat. ${ }^{i}$ Die vorliegende Untersuchung versteht sich

' E.g. J. Bremmer (1994), S. 76-78 und S. 84-86; A.C. Brumfigld (1981); Ph. Bruneau (1970); W. BuRKert (1977), S. 247-251, 365-370 und 426-432 = id. (1985), S. 159-161, S. 242-246 und S. 285-290; W. BURKER' $(1987)=i d .(1990)$; K. CUNTON (1996); H.P. FOLEY (1994); N. DEMAND (1994); M. DETIENNE $(1979)=i d .(1989) ;$ B.C. DieTriCh (1974) sowie (1986); F. GRAF, DNP 3 (1997), Sp. 420-426, s.v. Demeter; id., DNP 8 (2000), Sp. 611-626, s.v. Mysteria und s.v. Mysterien; M.P. NiLSSON (1906), S. 311-362; id. (1967), S. $456-481$ und S. 653-678; H.W. PARKE (1977), S. 5572 und 82-88; H. PETERSMAnn, WS 99 (1986), S. 69-85 = id. (2002), S. 133-145; id., WS 100 (1987), S. $5-12=i d .(2002)$, S. $146-151$; $i d .(1987)$, S. 171-199 = id. (2002), S. 105-132; N.J. RICHARDSON (1974); E. SIMON (1983), S. 17-37; E. SIMON (1985), S. 91-117; H.S. VERSNEL (1993); J.J. WINKLER (1990); F.I. ZEITLIN (1981); ead. (1982).

2 V.L. Aravantinos, L. Godart, A. SACCONI (2001); Gesamtausgabe der bis Februar 2002 gefundenen mykenischen Dokumente von Theben: id., Foulles de la Cadmée III, Corpus des documents d'archives en linéaire B de Thèbes (1-433), Pisa/Rom, 2002.

3 V.L. ARAVANTINOS, L. GODART, A. SACCONI (2001), S. 188-194; früher schon im gleichen Sinne etwa L. GODART, A. SACCONI (2000), S. 19 f.

"E.g. P. Blome, „Die dunklen Jahnhunderte - aufgehellt", in J. LATACZ (ed.), Colloquium Rauricum, Band 2, Zweihundert Jabre Homer-Forscbung, Stuttgart/Leipzig, 1991, S. 45-60; S. DEGER-JALKOTZY, „Die Erforschung des Zusammenbruchs der sogenannten mykenischen Kultur und der sogenannten dunklen Jahrhunderte", ibid., S. 127-154 (mit reichhaltigen Literaturangaben S. 152-154); F. GSCHNITZER, "Vocabulaire et institutions: la continuité historique du deuxième au premier millénaire", in E. RISCH - H. MÜHLESTEIN (eds), Colloquium Mycenaeum, Actes du 6 colloque intemational sur les textes mycéniens et égéens (1975), Neuchâtel, 1979, S. 109-134= F. GSCHNITZER (2001), S. 106-131; id., Griechische Sozialgeschichte von der myenischen bis zum Ausgang der klassischen Zeit, Wiesbaden, 1981, S. 10-26 (Kapitel über die mykenische Zeit); J. LATACZ et al. (Katalogkonzeption), Begleilband zar Alsstellung "Troia-Traum und Wirklicbkeir" (Stuttgart/ Braunschweig/Bonn 2001/02), Stuttgart, 2001; M.L. WEST, ,The Rise of the Greek Epic“, JHS 108 (1988), S. 151-172; aus dem religionswissenschaftlichen Bereich e.g. R. LAFFINEUR R. HÄGg (eds), Polnia, Deities and Religion in the Aegean Bronze Age, Proceedings of the 8th International Aegean Conference Göleborg, Göteborg University, 12-15 April 2000, Liège, 2001 (Aegaetm, 22), passim. 
als Beitrag zu einem besseren religionshistorischen Verständnis der eindeutig tief in vorgeschichtlichen Zeiten wurzelnden Demeter und ihrer Tochter.

Im 1. Jt.v.C. gehören Demeter und Kore zu den populärsten Gottheiten und geniessen in der ganzen griechischen Welt kultische Verehrung. Am bekanntesten sind dabei einerseits das Thesmophorienfest, das von sämtlichen griechischen Festen die grösste Verbreitung aufweist, ${ }^{5}$ und andererseits die Mysterien von Eleusis, die gerade umgekehrt auf diese eine Ortschaft beschränkt sind. ${ }^{6}$ Es sind dies die einzigen Demeterkulte, die sowohl reichhaltig dokumentiert als auch nachweisbar aus voralphabetischen Zeiten ererbt sind. Sie sollen hier im Zentrum des Interesses stehen.

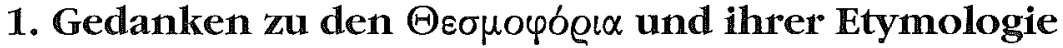

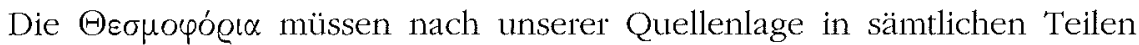
Griechenlands gefeiert worden sein, ${ }^{7}$ was sonst von keinem einzigen der zahlreichen griechischen Feste gilt und allein schon für ein hohes Alter des Thesmophorienfestes spricht - sekundäre Ausbreitung im historischen Griechenland ist undenkbar." Genaueres ist uns von den athenischen

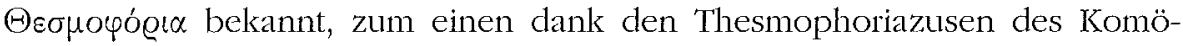
diendichters Aristophanes und den zugehörigen antiken Kommentaren, zum anderen auch dank einem viel diskutierten Lukianscholion." Es handelt sich um ein jedes Jahr im Herbst ${ }^{10}$ durchgeführtes dreitägiges Fest, an dem nur Frauen teilnehmen durften. Nachdem lebendige Ferkel in unterirdische Grotten $(\mu \varepsilon ́ \gamma \alpha \varrho \alpha)$ geworfen worden waren, brachten bestimmte Frauen die Reste dieser Tiere wieder herauf und legten sie zusammen mit anderen

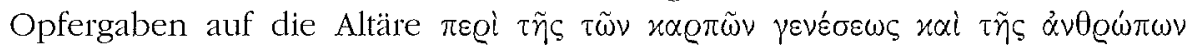

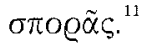

Die Bezeichnung $\Theta \varepsilon \sigma \mu o \varphi o ́ \varrho l \alpha$ ist auf den ersten Blick vergleichsweise

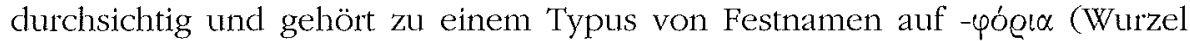
*pher- 'tragen'), dem eine ganze Anzahl Festbezeichnungen angehören ${ }^{12}$ und der bereits im 2. Jt. bezeugt ist. ${ }^{13}$ Unklar ist jedoch, was wir uns unter $\theta \varepsilon \sigma \mu o-$

5 L.R. FARNELl (1906), S. 328ff;; M.P. NiLSSON (1906), S. 313-325.

${ }^{6}$ E.g. W. Burkert (1977), S. 426-432 = W. BURKERT (1985), S. 285-290.

7 Vgl. loc, cit. in der Anm. 5.

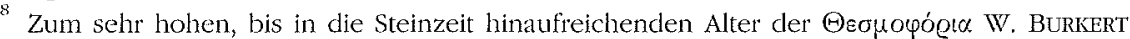
(1977), S. $369=i d$. (1985), S. 244f; H.W. Parke (1977), S. 82; H. Petersmann (1987), S. $179=i d$. (2002), S. $112 \mathrm{f}$.

"Schol. Luc. S. 276, 2ff., ed. Rabe; vgl. L. Deubner (1932), S. 50-60; M.P. Nilsson (1967),

S. 463; H.W. PARKE (1977), S. 82-88.

10 W. BURKerT, l.c. (Anm. 8); C. TRÜMPY (1997), S. $175 \mathrm{f}$.

1 Schol. Luc. S. 276, 14f., ed. RABE.

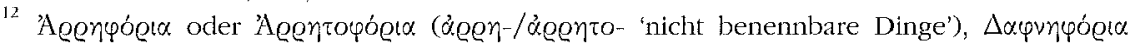

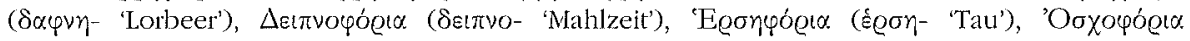

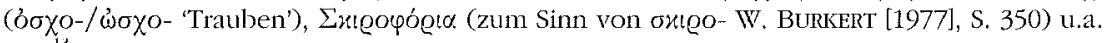

is te-o-po-ri-ja (KN Ga 1058, Od 696: /theophória/) 'Gott- bzw, Götter-Trage-Fest'. 
vorzustellen haben. Angesichts der Existenz des Begriffs $\theta \varepsilon \sigma \mu o ́ s ~ ' S a t z u n g '$, 'Gesetz' $z^{14}$ ist die Gleichsetzung von $\theta \varepsilon \sigma \mu o_{-}$in $\Theta \varepsilon \sigma \mu o \varphi \rho ́ \varrho \iota$ mit diesem

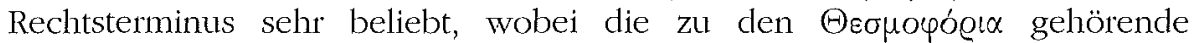

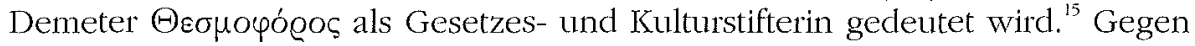
eine derartige Annahme spricht aber schon, dass die Festnamen des Typs $\mathrm{x}$ pó@ı in allen anderen Fällen Vorderglieder aufweisen, die konkrete Gegen-

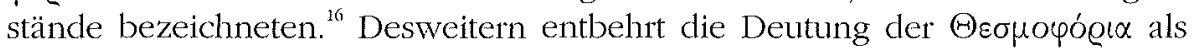

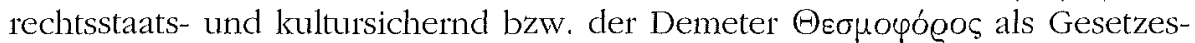
und Kulturstifterin jeglicher Grundlage in unserem Quellenmaterial. Weder Demeter noch Kore stehen sonst mit der Gesetzgebung in Verbindung.

Die $\Theta \varepsilon \sigma \mu о \varphi o ́ \varrho \iota \alpha$ wurden auch mit 'Hingelegtem' (Wz. * $d^{h} e b_{1^{-}}$, vgl. $\tau(\theta \eta \eta \mu)$ in Verbindung gebracht; gemeint wären die Ferkel und andere Opfergaben. ${ }^{17}$ Einzuwenden ist hier erstens, dass die Ferkel nach unserer Ueberlieferung nirgendwohin gelegt, sondern in die $\mu \varepsilon ́ \gamma \alpha \varrho \alpha$ geworfen wurden (ausgedrückt durch die Verben éi $\pi \tau \varepsilon v \nu$ und $\beta \alpha \dot{\alpha} \lambda \lambda \varepsilon \iota v)$. Aber auch die anderen Opfergaben, die man auf die Altäre legte, können mit dem postulierten $\theta \varepsilon \sigma \mu o-$ 'Hingelegtes' nicht gemeint sein. 'Hingelegtes-Trägerinnen' ergibt jedenfalls im Zusammenhang der $\Theta \varepsilon \sigma \mu o \varphi o ́ \varrho \iota \alpha$ keinen plausiblen Sinn. Zweitens widersetzen sich auch die Wortbildungsregeln einer Deutung von $\theta \varepsilon \sigma \mu o-$ als 'Hingelegtes'.

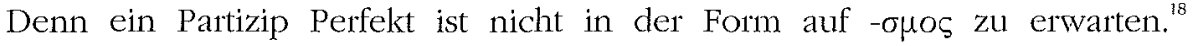
Sowohl gegen $\theta \varepsilon \sigma \mu o-$ 'Satzung', 'Gesetz' als auch gegen $\theta \varepsilon \sigma \mu 0_{-}$'Hingelegtes' spricht nun aber entscheidend ein Argument dialektologischer Art, das, soweit ich sehe, niemals Beachtung gefunden hat:

1.j Dazu F. GSCHNITZER (1997) = id. (2001), S. 261-268.

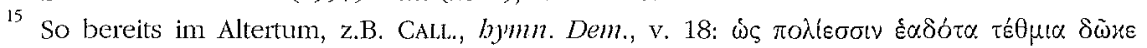
(scil. Demeter); DioD., V, 5, 2; SERV., ad Aen. IV, 58. In der modernen Forschung z.B. p. Chantraine (1968ff.), Bd. 1, S. 432, s.v. $\theta \varepsilon \sigma \mu o ́ s: ~ „ \theta \varepsilon \sigma \mu o \varphi b ́ o \varsigma$ 'qui apporte des lois, qui civilise” épithète de Déméter"; H. Jeannatre, Couroi et courètes, Lille, 1935, S. 305f; M. Jost (1985), S. 323f.; LSJ, S. 795, s.v. Oeouopógog; H. Petersanann (1987), S. $191=i d$. (2002), S. 124; U. v. WILAMOWITZ-MOELLENDORFF, Der Glaube der Hellenen I, Berlin, 1931, S. 208.

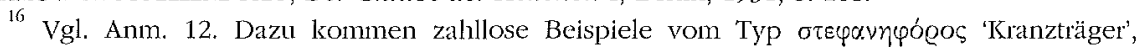

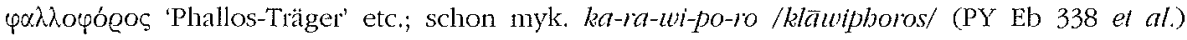

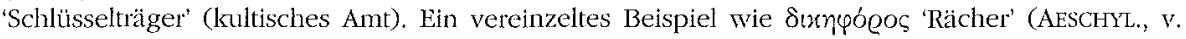
525 und 1577; Cboeph., v. 120) vermag das Argument nicht zu entkräften. Vgl. auch H.W. PAkKE

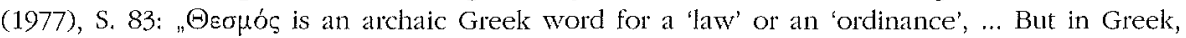
unlike English, the verb 'carry' is not normally used in such a connection. Also Demeter does not properly appear to be concerned with legislation. Hence, starting with Frazer, some modern scholars had proposed that ... the $\theta \varepsilon \sigma \mu o$ must be some material objects which could be physically carried. Here there is a possible object which is known from descriptions of the ritual of the Thesmophoria. The only difficulty is that there are few examples to suggest how the word $0 \varepsilon \sigma \mu$ of acquired this meaning."

17 So schon V. EHRENBERG (1921), S. 111f; w. BuRKERT (1977), S. 367 = id. (1985), S. 243; L. Deubner (1932), S. 45; M.P. Nilsson (1967), S. 464 (mit Anm. 4); E. Simon (1983), S, 19.

${ }^{18} \mathrm{Zu}$ den Funktionen des Suffixes $-\mu o-/-\theta \mu o-/-\sigma \mu o-$ P. Chantrains (1933), S. 132-147; E. SCHWYZER (1939), S. 491-493. 


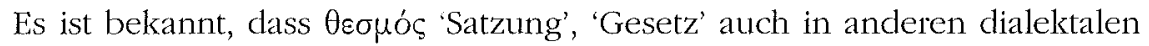
Varianten auftritt, nämlich in den Formen $\tau \varepsilon \theta \mu o ́ \varsigma$ und $\theta \varepsilon \theta \mu o ́ c .{ }^{19}$ All diesen Formen wird einhellig die Wurzel $* d^{b} e b_{1}$ - des Verbums $\tau i \theta \eta \mu$ isetzen', 'stellen', 'legen' zugrunde gelegt. Dabei wird zum Teil mit einer Grundform * $d^{b}$ ed $d^{b}$ mo- gerechnet, zum Teil aber auch damit, dass diese Wurzel je nach Dialektgebiet einmal mit - $\sigma \mu \rho \varsigma$ und einmal mit $-\theta \mu o \varsigma$ erweitert sei. Die Hypothese einer Ausgangsform * $d^{h} e d^{h} m^{\prime}$-, die die reduplizierte Form der Wurzel enthielte, ist deswegen unglaubwürdig, weil sie ohne morphologische Parallele bliebe. Denn nicht nur ist im Griechischen Nominalbildung mittels Reduplikation nur vereinzelt greifbar; ${ }^{20}$ zudem gibt es kein einziges derartiges Beispiel, das wie ${ }^{*} d^{b} e d^{b}-m_{0}$ - (mit Suffix - $\mu_{-}$) ) suffigiert wäre. Ferner ist auch die unter der Annahme eines zugrundeliegenden $* d^{b} e d^{b}$ mo-vorauszusetzende Lautentwicklung $*_{-} d^{b} m->-\sigma \mu$ - problematisch, da sich der Dental in einer derartigen Lautkombination normalerweise nicht zu einem $-s$ - entwickelt. ${ }^{21}$

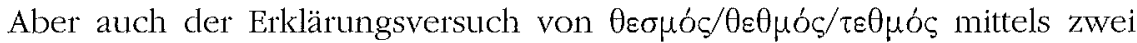
verschiedener Suffixe $\left(-\theta \mu 0_{-}\right.$und - $\left.\sigma \mu o_{-}\right)$befriedigt nicht. Denn unterschiedliche Suffixation gibt kaum je eine plausible Etklärung von Dialektvarianten ab, insbesondere dann nicht, wenn es sich, wie in unserem Fall, um ins 2. Jt. hinaufreichende Dialektmerkmale handeln müsste (im 1. Jt. sind $-\theta \mu o-$ und

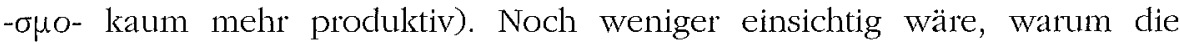
unterschiedlichen Dialekte sich teils für - $\sigma \mu \circ \varsigma$ und teils für $-\theta \mu \circ \varsigma$ entschieden haben sollten. ${ }^{22}$

Die sprachlich sauberste Lösung wäre ohne Zweifel eine lautgesetzliche Entwicklung von älterem $-\theta \mu \circ \varsigma$ zu ion. $-\sigma \mu \circ \varsigma$ der historischen Zeit (während der umgelkehrte Weg $-\sigma \mu o \varsigma>-\theta \mu o \varsigma$ im Griechischen unmöglich ist). Ich konnte aber nur ganz vereinzelte Beispiele finden, die diese einfachste Erklärung stïtzen könnten. ${ }^{23}$

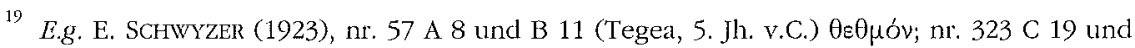

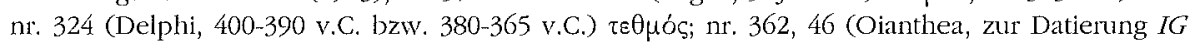

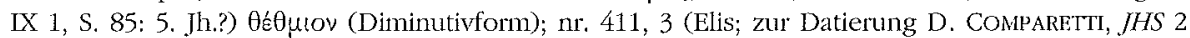

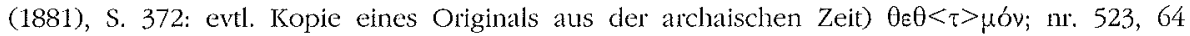
(Orchomenos; $222-220$ v.C.) $\theta \varepsilon \theta \mu i ́ \bar{o}$. Die ebenfalls auftretende Form $\tau \varepsilon \tau \theta \mu o ́ s$ ist als graphische Variante zu verstehen (lokr., 525-500 v.C.: $I G I X^{2} 1$, nr. 609; in dieser Inschrift findet sich daneben auch die Form $\tau \varepsilon \theta-$-).

20 P. Chantraine (1933), S. 6. Das einzige Beispiel für eine eindeutig voreinzelsprachliche

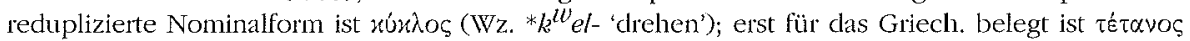

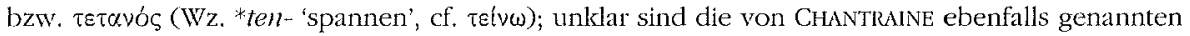

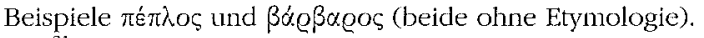

${ }^{21}$ M. LEjEUNE (1972), S. 76f., \$ 66; A.L. SIHLER (1995), S. 208, $\$ 221$

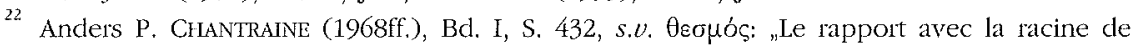
ti $\theta \eta \mu$, etc., est évident. ... il n'y a pas lieu de poser une forme à redoublement *dhe-dbmo- où

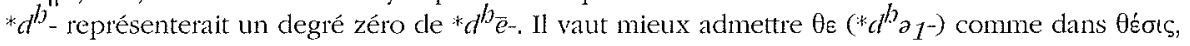
avec des suffixes $-\sigma \mu \circ \varsigma_{\text {(propre au grec) ou }-\theta \mu o \varsigma, \ldots . .}$

${ }^{23}$ F. GSCHNITZER weist mich u.a. auf vereinzelt belegtes ion. ఏvouós neben nomalem (auch

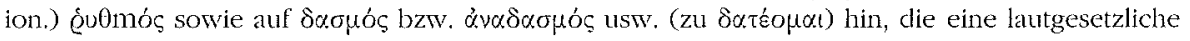


Wie dem auch sei, weder die Festbezeichnung $\Theta \varepsilon \sigma \mu o \varphi o ́ g \iota \alpha$ (bzw. der

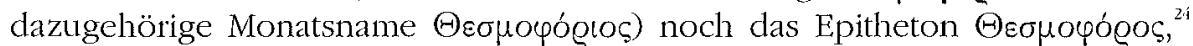
die gerade auch für dasjenige Dialektgebiet besonders gut bezeugt sind, in dem für 'Satzung', 'Gesetz' die Variante $\tau \varepsilon \theta \mu o ́ \varsigma$ (bzw. $\theta \varepsilon \theta \mu o ́ c$ oder $\tau \varepsilon \tau \theta \mu o ́ c)$ heimisch ist, sind jemals in einer anderen lautlichen Form als in der Form $\Theta \varepsilon \sigma \mu o-$ belegt - trotz ihres ausgesprochen zahlreichen Auftretens schon in vorhellenistischen Zeiten, in denen die typischen Dialektmerkmale noch gut bewahrt sind. Das aber heisst unmissverständlich, dass die $\Theta \varepsilon \sigma \mu о \varphi o ́ \varrho ı \alpha$ etymologisch von $\theta \varepsilon \sigma \mu \delta \varsigma / \tau \varepsilon \theta \mu o ́ s$ 'Satzung', 'Gesetz' zu trennen sind.

Nun gibt es ein weiteres wichtiges Wort, das ebenfalls mit der Wurzel von $\tau i \theta \eta \mu \iota\left({ }^{*} d^{h} e b_{1}\right)$ in Verbindung gebracht wird, wenn auch dessen Zuordnung zu $\tau i \theta \eta \mu$, anders als im Falle von $\theta \varepsilon \sigma \mu o ́ s$, umstritten ist: $\theta \varepsilon o ́ \varsigma$ „Gott“ (myk. te-o /theós/ $)^{.5}$ In bezug auf die Bedeutung lässt sich $\theta \varepsilon o ́ s$ als Ableitung der Wurzel ${ }^{*} d^{b} e b_{1-}$ durchaus zufriedenstellend erklären. Es müsste sich ursprünglich um etwas Aufgestelltes oder Hingelegtes handeln, um ein Götterbild bzw. eine Statue. Dabei wird freilich ein ernst zu nehmendes Problem ausser Acht gelassen, nämlich der Makel eines unerklärten $*_{-s-}$. Denn der Hiat zwischen den beiden Vokalen von $\theta \varepsilon o ́ s$ muss zwingend mit einem ursprünglich vorhandenen intervokalischen $*_{-s-}(>$ schon myk. $-b-$ ) erklärt werden, was auch niemand bestreitet. ${ }^{26}$ Die früheste rekonstruierbare Vorstufe des Wortes $\theta \varepsilon$ ć muss $* d^{b} b_{1}$ sos gelautet haben (um ca. 2000 v.C. und vielleicht auch noch später, vor den Entwicklungen von $* d b->* t b-, *_{-} b_{1}$ - (Laryngal) $>-e$ - und von intervok. $*_{-S-}>-b$-, die alle auf den myk. Täfelchen um 1200 abgeschlossen sind). $\mathrm{Zu} * d^{b} b_{1}$ sos passen auch lat. fänum ( $<* d^{b} b_{1}$ snom), lat. festus $(<$ $* d^{b} b_{1}$ stos oder $<* d^{b} e b_{1}$ stos ?), lat. feriae $\left(<* d^{b} e b_{1} s^{-i b_{2}}\right)$, osk. fiisnu, und armen. di-k', nicht nur aus lautlicher Sicht, ${ }^{27}$ sondern auch insofern, als sie allesamt eine Wurzel $* d^{b}(e) b_{1}{ }^{-}$zu enthalten scheinen und der kultischen

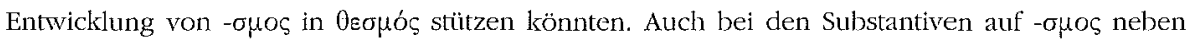
Verben auf $-\zeta \omega<*$-djo vermutet F. GsCHNITZER teilweise ein lautgesetzliches Entstehen von $-\sigma \mu-<$

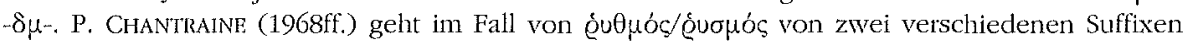

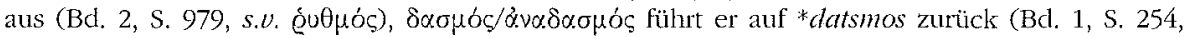

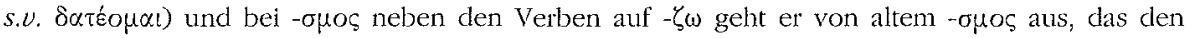
vorausgehenden Dental verdrängt hätte (P. CHANTRAine [1933], S. 138). Die Belege lassen jedenfalls kaum eine systematische Lautentwicklung $-\tau \mu-/-\theta \mu-/-\delta \mu->-\sigma \mu$ - in einem bestimmten Dialektgebiet erkennen. Eine neue Bearbeitung des gesamten relevanten Materials wäre nötig, um die Frage zu klären.

24 In Arkadien trägt Demeter auch den Beinamen @éouıo̧ (Paus., VIII, 15, 4), der evidenterweise eine adjekivische Ableitung desselben Wortes darstellt, das in $\Theta \varepsilon \sigma \mu \circ-\varphi \circ Q-$ vorliegt. Vgl. zu $\Delta \eta \mu \dot{n} \tau \eta$ e $\Theta \varepsilon \sigma \mu l \alpha$ M. Jost (1985), S. 322-324.

${ }^{25}$ Vgl. P. Chantraine (1968ff.), Bd. 1, S. 430, s.v. Oeós (,... Finalement l'ensemble reste incertain."). Zugunsten einer Verbindung von $\theta \varepsilon o ́ s$ mit $\tau i \theta \eta \mu$ äusserte sich zuletzt H. PETERSMANN, "Beobachtungen zu den Appellativen für "Gott", in K.-F. KrafT - E.-M. Lill - U. SchwaB (eds), Trinue, Sudien zur Sprachgeschichte und Literaluwissenschaft, Gedächtnisbuch fïr E. STuTz, Heidelberg, 1992, S. 139-141 = id. (2002), S. 20f; id., Ktema 15 (1990), S. $80=i d$. (2002), S. 27. (Bei M. MAyrhofer [1986], S. 127, zitiert von PETEIsSMann, ist freilich nicht die Rede davon, dass

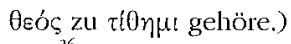

${ }_{26}^{26}$ Vgl. e.g. P. Chantraine, l.c.. in der vorhergehenden Anm.

${ }^{27}$ Dazu M. MAYrhofEr, l.c. (Anm. 25). 


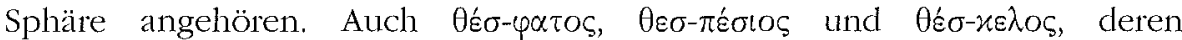
Vorderglied $\theta \varepsilon \sigma^{-}$üblicherweise mit $\theta \varepsilon o ́ s$ in Zusammenhang gebracht wird, fügen sich gut ins Bild: Die Existenz einer Wurzelgestalt $* d l^{b}(e) b_{1} s^{-}$, mit welcher Bedeutung auch immer, ist demnach vergleichsweise gut gesichert, eine Erweiterung der Verbalwurzel $* d^{b}(e) b_{1^{-}}$'setzen', 'stellen' durch -s- aber (abgesehen vom hier zur Diskussion stehenden, ad boc vermuteten $* d^{b} e b_{1-s-}$ ) nicht nur im Griechischen und im Lateinischen, sondern auch im Germanischen und in allen anderen verwandten Sprachen unbekannt. ${ }^{28}$ Deswegen scheint es mir methodisch richtig, von einer von *db $(e) b_{1}$ - 'setzen', 'stellen' unabhängigen Wurzel $* d^{h}(e) b_{1}{ }^{\text {- }}$ auszugehen, deren Bedeutung angesichts ihres ausschliesslich kultischen Vorkommens im Kult zu suchen und etwa mit 'heiligen' oder 'dem Bereich der Götter zuordnen' u.ä. ${ }^{29}$ zu umreissen ist." $\Theta \varepsilon o ́ c$ 'Gott' wüirde damit ursprünglich eine Thematisiesung von $* d^{b}(e) h_{1} s^{-}$ darstellen und die Gottheit als 'Heiliges bewirkend' (aktivisch) und gleichzeitig als 'göttlich verehrt' bzw. 'Heiliges empfangend' (passivisch) charakterisieren, ${ }^{31}$ lat, fänum ist ohnehin das Heiligtum, ebenso osk. físnu, lat, festus wäre zu verstehen als 'geheiligt' wie als 'Heiliges bewirkend', lat. fêriae (ursprünglich eine feminine Substantivierung einer Ableitung auf -io-? s. oben, Anm. 29) als 'geheiligte Zeitperiode', und armen. $d i-k$ ' bedeutet schliesslich eindeutig 'Götter'. Die (schwundstufige) Form $\theta \varepsilon o ́ c<* d d^{b} b_{1} s$-os ist zwar aussergewöhnlich, da thematische Bildungen normalerweise die o-Vollstufe aufweisen. ${ }^{32}$

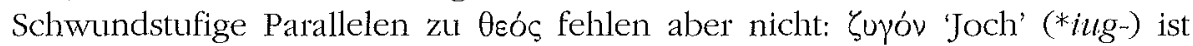
nach Ausweis der formalen Parallelen im Lat. (iugum), im Sanskrit (yugám) und im Germanischen eine besonders altertümliche schwundstufige Bildung und mag die Vermutung nahelegen, wir könnten es bei $\theta \varepsilon o ́ c ̧$ trotz seines Fehlens in anderen idg. Sprachen mit einem besonders alten Wort zu tun haben.

Nach alledem scheint es mir nun aber äusserst naheliegend, den Bestand-

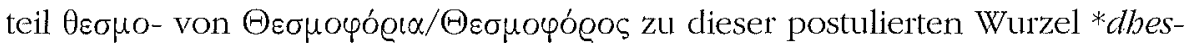
'heiligen' zu stellen. Auf der lautlichen Ebene ergibt sich damit freilich eine neue Schwierigkeit: Altes intervokalisches -sm- entwickelte sich je nach

${ }^{28}$ Die Wurzel $* d^{b}(e) b_{1} s$ - mag in voreinzelsprachlichen Zeiten, d.h. im 3. Jt. (oder früher?), durch falsche Morphemabgrenzung, etwa bei $* d^{b} b_{1} s-s q^{l t}$ (> $\theta$ ectécotos), zustande gekommen sein und letztlich doch ursprünglich zu $* d^{h}(e) b_{1^{-}}$'setzen', 'stellen' gehören. Für unsere Zwecke ist dies aber ohne Belang. Denn in den mit ${ }^{*} d^{b}(e) h_{1} s$ - gebildeten Wörtern der Einzelsprachen ist keinerlei Bezug zu einer allfälligen Vorgängerwurzel nachweisbar.

29 Nach den Zeugnissen scheint diese Wurzel nur nominal gebraucht worden zu sein; die Ableitungen auf -no- und to- im italischen Bereich (vgl. M. LEUMANN, Lateinische Laut- und Formenlehre, München, 1977, S. 320 und 333f.) könnten aber eine Verbalwurzel fortsetzen. Ferner weist mich R. WACHTER (Basel) noch darauf hin, dass feriae eine Substantivierung eines ebenfalls

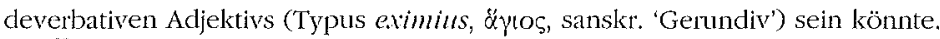

30 Vgl. auch ved, dbis-niya- 'Götter geneigt machend' (so MAYRHOFER, loc. cit, in der Anm. 25). Die Bedeutung von dbis- ist jedoch nicht gesichert, s. M. MAYRHOFER (1963), S. 103, s.v. abișánă (Name einer Göttin!).

${ }^{31} \mathrm{Zu}$ den Verwendungsweisen des Themavokals -o- P. CHANTRANE (1933), S. 6-17; E. SCHWYZER (1939), S. 457-459.

32 Vgl. l.c. in der vorhergehenden Anm. 
Dialektgebiet entweder zu $-\mu \mu$-oder zu $-\mu$ - mit ersatzgedehntem vorherge-

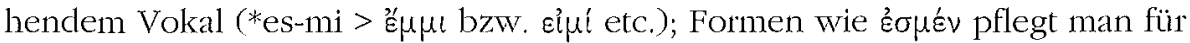
analog, nicht lautgesetzlich zu halten. ${ }^{33}$ Im Falle von Bildungen auf - $\sigma \mu o \varsigma$ (und

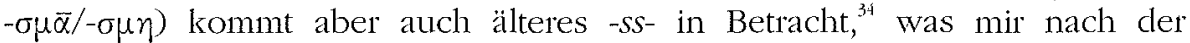
Identifikation der Wurzel *dhes- das einfachste zu sein scheint: *dhes-smos bzw. griech. *thes-smos hätte sich zu einer nicht genauer präzisierbaren Zeit vor den alphabetischen Zeugnissen zu $\theta \varepsilon \sigma \mu o ́ s$ vereinfacht. Auf jeden Fall darf das Suffix $-\sigma \mu o$ s schon für prähistorische Zeiten vorausgesetzt werden, nach den Beispielen zu schliessen, wo mit einem geschwundenen $s$ dieses Suffixes zu rechnen ist. ${ }^{35}$

Aber auch auf der inhaltlichen Ebene ist die hier vorgeschlagene Etymolo-

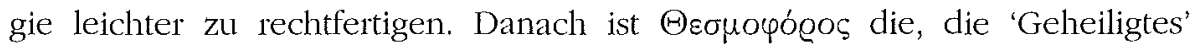
trägt, i.e. die Reste der Ferkel und andere Opfergaben, die während der $\Theta \varepsilon \sigma \mu о \varphi o ́ g l \alpha$ eine Rolle spielten, vielleicht auch das neue Saatgut, alles Dinge, die nach unseren Zeugnissen während des Festes zu tragen oder zu bringen waren, ${ }^{36}$ Nicht auszuschliessen ist, dass sich die beiden Homonyme $\theta \varepsilon \sigma \mu o_{-}$ 'Heiliges' und $\theta \varepsilon \sigma \mu o ́ s$ 'Satzung', 'Gesetz' gegenseitig beeinflussten: 'Gesetz' mag mit 'Heiligem' assoziiert worden sein und umgekehrt.

Es stellt sich nun aber auch die Frage, ob die oben erwähnte, merkwürdige Tatsache, dass neben ion. $\theta \varepsilon \sigma \mu o ́ s ~ ' G e s e t z '$ Dialektvarianten mit - $\theta \mu$ existieren, nicht am ehesten dadurch erklärt werden könnte, dass nur die

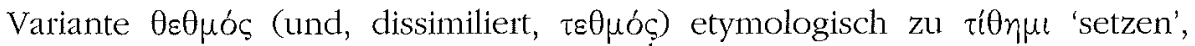
'stellen' gehört (schwundstufige Wurzel ${ }^{*} \mathrm{~d}^{\mathrm{h}} \mathrm{eh}_{1}$ - und bekanntes Suffix $-\theta \mu_{\mathrm{O}}{ }^{37}$ ). Die ionische Lautung $\theta \varepsilon \sigma \mu o ́ \varsigma$ wäre dagegen durch eine sekundäre Verwechslung mit $\theta \varepsilon \sigma \mu o-$ 'Heiliges' zustande gekommen, vielleicht erst in nachhomeri-

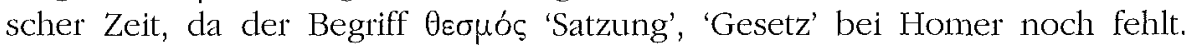
Eine gewisse Nähe von $\theta \varepsilon \theta \mu o ́ s ~ ' G e s e t z '$ zum göttlichen Bereich und die Häufigkeit der Festbezeichnung $\Theta \varepsilon \sigma \mu o \varphi o ́ @ ı \alpha$ bzw. der Demeter $\Theta \varepsilon \sigma \mu о \varphi o ́ \varrho o \varsigma$ - die angesichts des Fehlens einer durchsichtigen Wurzel *thes- 'heilig' in der Umgangssprache des 1. Jt. für die Sprecher ein nicht mehr durchschaubares

33 M. LEJEUNE (1972), S. 122f., $\$ 116$; A.L. SIHLER (1995), S, 208, $₫ 221$; speziell zum Suffix

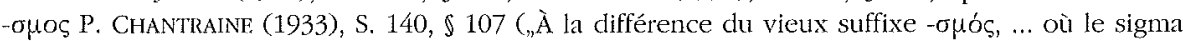
est phonétiquement tombé en ionien-attique devant $\mu$, le groupe $\sigma \mu$ est toujours maintenu. D'autre part le sigma provient évidemment, dans presque tous les cas, du système verbal ... La catégorie des dérivés en $-\sigma \mu o \varsigma$ étant productive, la finale - $\sigma \mu o ́ s$ se trouve impliquée dans quelques mots, même si la flexion verbale ne présente aucun élargissement en sigma. ... $\theta \varepsilon \sigma \mu o ́ s$

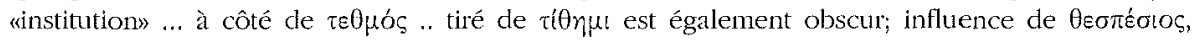

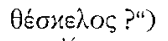

31 Vgl. E. SCHWYZER (1939), S. 311.

35 Beispiele bei P. Chantraine (1933), S. 138.

36 Weniger wahrscheinlich scheint mir, das 'Heilige' mit Fruchtbarkeit zu identifizieren. Die $\Theta \varepsilon \sigma \mu o \varphi o ́ g o s$ ist wohl diejenige, die den Menschen die Fruchtbarkeit der Äcker, der Tiere und der Menschen garantieren soll, aber die Beobachtung, dass - póos sonst nur von Konkreta gebraucht wird, spricht zum vomherein gegen jede Deutung von unserem $\theta \varepsilon \sigma \mu_{0}$-als Abstraktum (s. oben).

${ }^{37} \mathrm{Zu}-\theta \mu \mathrm{o}$-oben, Anm. 18. 
Vorderglied aufwiesen - wären dann für die ionische Neugestaltung mitverantwortlich gewesen. ${ }^{38}$

Im übrigen findet sich der früheste Beleg für $\theta \varepsilon \sigma \mu o ́ s$ in der Odyssee (XXIII, 296), und da passt 'Satzung', 'Gesetz' sicher nicht, ${ }^{39}$ während 'heiliger Ort' kaum Schwierigkeiten bereiten würde.

Auch in nachhomerischen Zeiten gibt es verschiedene Stellen, wo 'Satzung', 'Gesetz' für $\theta \varepsilon \sigma \mu o ́ s$ nicht gemeint sein kann. Am wichtigsten ist in unserem Zusammenhang das Epigramm IG II-III² 3, nr. 3639 (Eleusis, ca. 170 n.C.), wo von den $\dot{\alpha} \varrho \varrho \eta ́ \tau \omega \nu ~ \theta \varepsilon ́ \sigma \mu l \alpha$ als den wesentlichen Inhalten des eleusinischen Kultes die Rede ist. ${ }^{\text {i0 }}$

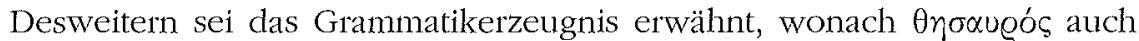
mit $\theta \varepsilon \sigma \mu o ́ \varsigma$ ausgedrückt werden könne, so bei Anakreon. ${ }^{41}$ Nach unseren obigen Ausführungen ist es verlockend, auch $\theta \eta \sigma-\alpha v \varrho o ́ s$ mit *thes- in Verbindung zu bringen. Mit Langvokal ist *thes jedenfalls in lat, feriae bezeugt, während lat. festus bezüglich der Wurzelquantität nicht eindeutig bestimmbar

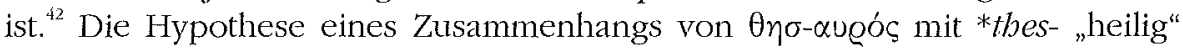
kann aber auch noch gestützt werden. Ein wiederum in den eleusinischen

38 Bemerkenswert sind in diesem Zusammenhang folgende Ausführungen V. EHRENBERGS (1921) zum Begriff $\theta \varepsilon \sigma \mu o ́$ (S. 109f.): "Gehen so konkrete und abstrakte Bedeutung bei Pindar und Aischylos nebeneinander, so ist doch e i n e s durchgängig“ (ibid. Anm. 3: „Ebenso bei

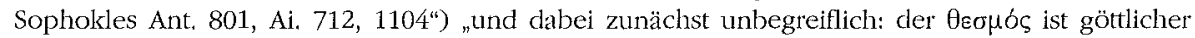
Herkunft, ein Gott oder ein Heros hat ihn gegeben. Es scheint unerklälich, wie das von menschlichen Richtern und Gesetzgebern Gesetzte einen religiösen und heiligen Charakter annehmen kann. Da ist zunächst der Haupteinwand gegen die menschliche Herkunft des $\theta \varepsilon q u o ́ s ~ u ̈ b e r h a u p t$

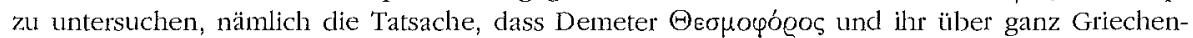

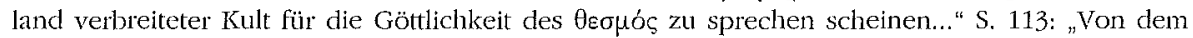
konkreten $\theta \varepsilon \sigma \mu o \zeta \varsigma$ des Kults zu irgendwelcher 'Satzung' führt kein Weg. Aber man könnte glauben, auch wenn eine Entwicklung des kultischen $\theta \varepsilon \sigma \mu o ́ \varsigma$ sich nicht annehmen lässt, dass doch die Abstrahierung und Heiligung des juristischen Begriffs sich unter dem Einfluss der in Athen so mächtigen Demeterreligion vollzieht ..."

${ }^{39}$ Es geht um Odysseus und Penelope, die nach der Wiedererkennung gern zum $\theta \varepsilon \sigma \mu \delta ́$ des

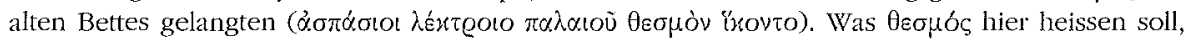
wird kontrovers diskutiert, vgl. A. HEUBECK, in A Commentary on Homers Odjssey III, Oxford 1992, S. 342 (ad loc.). Nach Heubeck hat $\theta \varepsilon \sigma \mu o ́ s$ hier vielleicht die nach ihm ältere Bedeutung 'Platz', 'Stelle'. Vgl. auch V. EHREnBerg (1921), S. 110, mit Literatur für die Auffassung, wonach

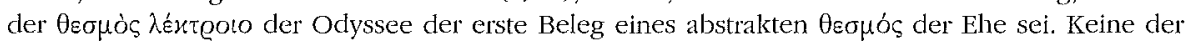
vorgebrachten Hypothesen vermag aber zu überzeugen.

40 Dazu G. Manganaro, ASAA 37f. (1959f.), S. 421-427, bes. S. 424-426. Vgl. auch M. DetienNE (1979), S. 199, Anm. 1, wonach die Bedeutung von $\theta \varepsilon \sigma \mu o ́ s$ im Wort $\Theta \varepsilon \sigma \mu o p o ́ \varrho \alpha$ nach dem

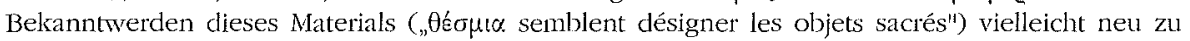
analysieren sei.

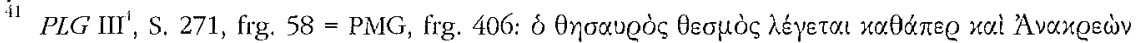

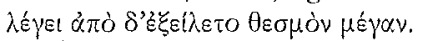

${ }^{42}$ Es sei hier auch an die äol. Form $\theta$ ńı

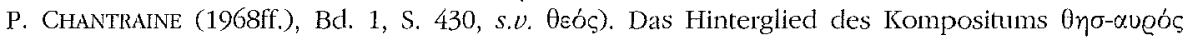

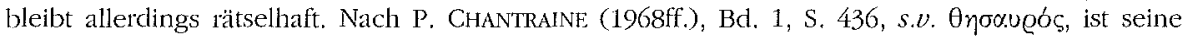
Etymologie ungeklärt. 
Kontext gehörendes kaiserzeitliches Epigramm enthält nämlich den Wortlaut:

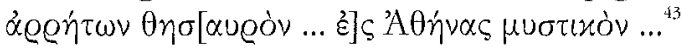

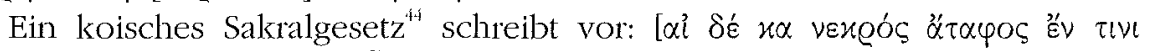

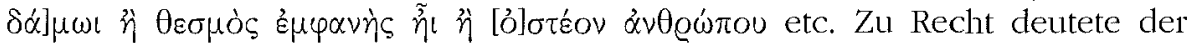
Herausgeber dieser Inschrift, R. Herzog, $0 \varepsilon \sigma \mu o ́ s$ hier als Grab. ${ }^{45}$

Es gibt auch noch eine ganze Serie weiterer Beispiele, in denen $\theta \varepsilon \sigma \mu o ́ s$ sehr auffällig mit dem göttlichen Bereich verknüpft ist, besonders oft bei den Tragikern. Ohne den geringsten Anspruch auf Vollständigkeit seien genannt:

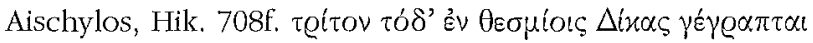

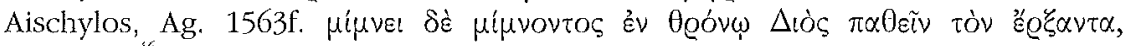
$\theta \dot{\varepsilon} \sigma \mu \iota v^{46} \gamma \alpha \dot{\alpha}$

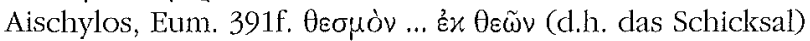

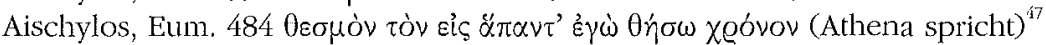

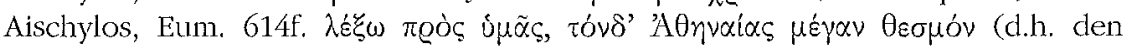
Areopag, Apollon spricht)

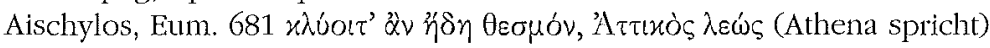

In all diesen Fällen lässt sich jedenfalls unter der Annahme, unsere Wurzel *thes- 'heiligen' liege zugrunde, die jeweilige Verwendungsweise von $\theta \varepsilon \sigma \mu o ́ s$ zwanglos erklären.

Umgekehrt wurden verschiedene chorlyrische Belege für den Begriff $\tau \varepsilon \theta \mu o ́ \varsigma$, die keinerlei Zusammenhang mit 'Gesetz' aufweisen, ${ }^{48}$ dahingehend interpretiert, dass dort der inhaltliche Zusammenhang mit $\tau \iota \theta \varepsilon v \alpha \iota$ deutlich sei. ${ }^{49}$ Nach unseren Ausführungen stellt sich aber die Frage, ob Pindar hier nicht in Wirklichkeit ein überliefertes altes, in den Dialekten seiner Zeit nicht mehr vorhandenes Wort $\theta \varepsilon \sigma \mu o ́ s$ „heiliger bzw. göttlicher Bereich“ o.ä. irrtümlich für die ionische Form von $\tau \varepsilon \theta \mu o ́ \varsigma ~ " G e s e t z "$ gehalten und deswegen in eine pseudo-dorische Form (eben $\tau \varepsilon \theta \mu o ́ c)$ gebracht hat.

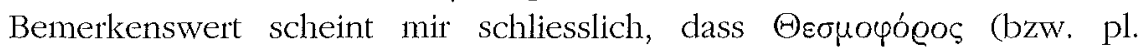
$\Theta \varepsilon \sigma \mu \circ \varphi o_{\varrho} \circ$ ) als Kultepitheton Demeters und Kores auftritt. Fast sieht es aus, als ob auch die beiden Göttinnen, zusammen mit den auserwählten Frauen,

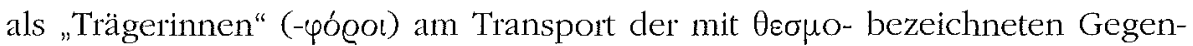
stände teilgenommen hätten, und man ist versucht, an das Phänomen der göttlichen Epiphanie ${ }^{50}$ zu denken, die in der minoischen Religion des 2. Jt. v.C., aus dem schliesslich die $\Theta \varepsilon \sigma \mu o \varphi o ́ \varrho \iota \alpha$ ererbt sein müssen, eine besonders

4.3 G. Manganaro, 1.c. (Anm. 40).

4 R. Herzog, Heilige Gesetze von Kos, Berlin, 1928, S. 22 (nr. 8, III. B, Z. 17).

${ }^{45}$ O.c. in der vorhergehenden Anm., S. 25 (dort noch zwei weitere Stellen, wo mit $\theta \varepsilon \sigma \mu o ́ s$ nach der Auffassung von Herzog vielleicht auch 'Grab' gemeint ist).

16 Oéoutos: Adj.- Ableitung von $\theta \varepsilon \sigma \mu o ́ s$.

$170 \varepsilon \sigma \mu o \dot{v}$... $\theta$ ńow ist zweifellos als Wortspiel zu verstehen.

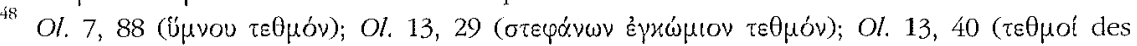

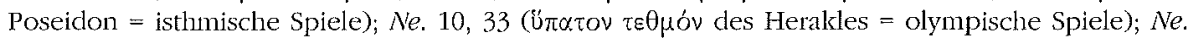

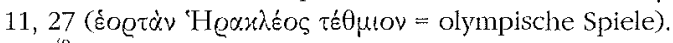

49) V. EHRENBERG (1921), S. 109.

50 F. GRAF, DNP 3, 1997, Sp. 1150f, s.v. Epiphanie. 
wichtige Rolle gespielt zu haben scheint. ${ }^{51}$ Für eine göttliche Epiphanie während des Thesmophorienfestes spricht auch die Beobachtung, dass nur die Göttinnen $\Theta \varepsilon \sigma \mu o \varphi o ́ \varrho o l ~ g e n a n n t$ werden können, während die am Kult teilnehmenden Frauen $\Theta \varepsilon \sigma \mu \circ \varphi \varphi^{\prime} \alpha \dot{\zeta} \zeta o v \sigma \alpha \iota$ heissen.

\section{Zum Verhältnis zwischen Demeter, Poseidon und der mykenischen ma-ka aus sprachwissenschaftlicher Sicht}

Auch das Theonym $\Delta \eta \mu \eta$ ine wird, zweifellos zurecht, einhellig als Kompositum aufgefasst. Ebenso unbestritten ist die Gleichsetzung des zweiten Gliedes - $\mu \dot{\eta} \tau \eta$ (bzw. im westgriech.-äolischen Dialektgebiet und in Arkadien

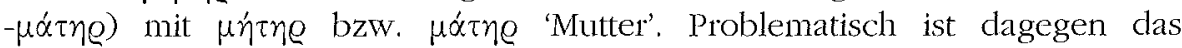
vorderglied $\Delta \eta-$ bzw. $\Delta \bar{\alpha}-$. Nach der traditionellen Deutung ${ }^{52}$ ist $\Delta \eta-/ \Delta \bar{\alpha}-$ bedeutungsmässig mit $\Gamma \tilde{\eta}$ 'Erde' gleichzusetzen, was zwar mit unüberwindbaren lautlichen Schwierigkeiten verbunden ist, jedoch schon wegen des ähnlichen Klanges von $\Gamma \tilde{\eta}$ und $\Delta \eta$ - verlockend wirkt und vor allem der Demeter als der Göttin des Getreides und der Fruchtbarkeit auch einen passenden Namen zu verleihen scheint: 'Mutter Erde'. ${ }^{53} \Delta \eta$ - bzw. $\Delta \bar{\alpha}$ - wurde oft der nicht-indogermanischen Sprachschicht der vorgriechischen Bevölkerung des 2. Jahrtausends v.C., und Demeter und ihr Kult dann letztlich diesem Substrat zugeordnet. Auch heute noch sprechen zum vornherein gewisse Indizien zugunsten einer nicht-indogermanischen Herkunft:

51 W. BURKER'T (1977), S. 78 = id. (1985), S. 40; R. HÄGG, „Die göttliche Epiphanie im minoischen Ritual“, MDAI 101 (1986), S. 41-62 (jeweils mit weiterführender Literatur); in unserem Zusammenhang ist HÄGGs Hinweis (S. 45) von besonderem Interesse, dass in der minoischen Kunst Götterdarstellungen, in denen Gottheiten und Menschen zusammen auftreten und die nach HÄGG in der Regel Kultszenen sein dürften, sehr zahlreich vorkommen.

${ }^{52}$ P. KRETSCHMER, „Demeter" ${ }^{44}$ WS 24 (1902), S. 523- 526 (Dā sei ein uralter Lallname der Erdgöttin); id., Glotla 17, 1929, S. 240 ( $D \bar{a}$ „vielleicht vorgriechisch").

53 Aus der reichhaltigen Literatur zur Etymologie des Theonyms Demeter seien noch zitiert: C.J. Rujgh, REG 80 (1967), S. 6f. (Nach RUJjGH ist die Deutung KrETsCHNERs nach wie vor einleuchtend; "d'autre part, il ne faut nullement exclure la possibilité qu'il s'agisse d'un nom d'origine préhellénique."); H. PETERSMANN (1987), S. 175-181 = id. (2002), S. 108-114 (S. $175 f$. bzw. S. 108f. findet sich eine Besprechung zahlreicher früherer Hypothesen; Demeter ist nach PETERSMANN etymologisch verwandt mit dem Toponym Dodona; im übrigen gehöre Demeter zu den ältesten vorindogermanischen Muttergottheiten). Vgl, auch W. BuRKERT (1977), S. 214, mit Anm, 2 = id. (1985), S. 136, mit Anm. 2 (auf der S. 402); P. Chantraine (1968ff), Bd. 1, S. 272, s.v. $\Delta \eta \mu \dot{\tau} \tau \eta \varrho$; H. FrISK (1960), S. 379f., s.v. $\Delta \eta \mu \dot{\eta} \tau \eta$ e. Zuletzt hat sich M. JANDA (2000), S. 257f., zur Etymologie von Demeter geäussext. Er versteht $D \bar{a}-m \bar{a} l \bar{e} r$ bzw. Dëmēerer als inclogemanische 'Gewiasser-Mutter'. (So vorher C. SCOTT LITILETON, „Poseidon as a Reflex of the Indo-European 'Source of Waters' God“, JIES 1 [1973], S. 423-440; M. NYMAN, „A pre-marine Vestige of $\theta \alpha \dot{\lambda} \alpha \sigma \sigma \alpha$ : An Etymological Proposal", Arclos 14 [1980], S. 51-78.) Diese Meinung krankt aber zum einen daran, dass Demeter auch in den frühesten greifbaren Zeiten nichts mit Gewässern zu tun hat, zum anderen auch daran, dass idg. * $d \bar{a}-\left(* d a H_{2}\right.$ ?) mit der Bedeutung 'Wasser' nur in einem vom griechischen Raum weit abgelegenen Gebiet plausibel gemacht werden kann und im Griechischen keinerlei sichtbare Spuren hinterlassen hat, sodass eine Deutung Demeters als ick. 'Gewässer-Mutter' ganz willkürlich ist. Ebenso willkürlich ist im übrigen JANDas idg. Etymologie des Namens Persephone, vgl. unten, Anm. 59, und C. TRÜMPY, Gnomon 75 (2003), S. 293. 
Erstens stellen die eng mit Demeter verbundenen $\mu$ vothel $\alpha^{5 / 3}$ ein solches

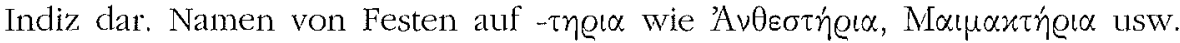
sind v.a. im ionischen Raum anzutreffen und finclen sich ähnlich bereits auf den mykenischen Tontäfelchen. 55 Dem Festnamen $\mu v \sigma \tau \hat{n} \varrho\llcorner\alpha$ liegt aber der Begriff $\mu$ ó $\tau \eta \varsigma$ „Einzuweihender" zugrunde, und anders als im Falle der

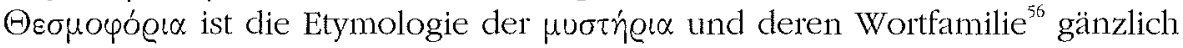
unbekannt, was ja für einen beachtlichen Teil des griechischen Wortschatzes

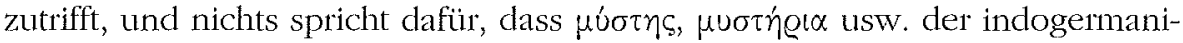
schen Schicht des Griechischen angehören.

Dasselbe gilt zweitens auch für die merkwürdigen Bezeichnungen Beú́ und Beırós, die im Kult von Eleusis im Rahmen einer hochtraditionellen Verkündigung eine Rolle spielen $^{57}$ und wiederum die Möglichkeit nichtindogermanischer Einflüsse unterstreichen.

Drittens treten im Zusammenhang mit Mysterien neben Demeter insbesondere auch Persephone und Dionysos in Erscheinung. ${ }^{58}$ Alle beide haben sich bisher - entgegen neueren, von idg. Wortmaterial ausgehenden sprachlichen Analysen $^{59}$ - einer überzeugenden etymologischen Deutung entzogen. M.E. sprechen aber schon die zahlreichen weder nach der Lautlehre noch nach dialektalen Kriterien erklärbaren Varianten, die es von Persephone wie von Dionysos gibt, ${ }^{60}$ für eine zumindest teilweise nicht-idg. Herkunft dieser Theonyme (in beiden Fällen muss es sich um Komposita handeln, wobei es denkbar ist, dass - wie im Falle von Demeter und Poseidon (s. unten) jeweils nur das eine Glied nicht-indogermanischer Herkunft ist ${ }^{61}$ ).

54 Zu den Mysterien unten, S. $30 f$.

55 (Sing.) re-ke-(e-)to-ro-te-ri-jo /lekbestrōerion/(PY Fr 343 und 1217) und to-no-e-ke-le-ri-jo thomobellaterion/ (PY Fr 1222).

56 Ihre Wurzel scheint bereits in myk. mu-jo-me-no, das man gewöhnlich als 'eingeweiht' versteht, bezeugt zu sein (PY Un 03 pa-ki-ja-si mu?-jo-me-no e-pi wa-na-ka-te a-pi-e-ke o-pi-te$\langle u\rangle-k e-e-\|)$.

57 Vgl. unten, S. 31f.

58 W. BuRKERT (1977), S. 413-451 = id. (1985), S. 276-304; id. (1972), S. 274-327; id. (1987) = id. (1990); H.P. FOLEY (1994), S. 67; M. JOST (1985), S. 434-436; N.J. RICHARDSON (1974), S. 27.

59 Zu Persephone M. JANDA (2000), S. 224-250 (idg. *perse-phat-ia 'die das/den (immer' wieder) Glänzende(n) hinüberbringt/hinüberbrachte'); H. PETERSMANN, Spracbe 32, 1986, S. 286$307=i d$. (2002), S. 152-169 (idg. "persa-gl'bon-eHz 'die von Feuer, Licht Ubervolle'). Zu Dionysos M. JANDA (2000), S, 258-260 (danach sei Dionysos ursprünglich 'der die Gewässer zum Fliessen bringt', im Anschluss an M. PETERs, Spracbliche Studien zum Fribgriecbiscben, Wien, 1989, S. 217-220 und S. 255-267, maschinenschriftl. Habilitationsschrift, zitiert von JANDA, non vidi)). Bei JANDA sind auf der S. 260, Anm. 577, verschiedene Forscher genannt, die Dionysos, der verbreitetsten Ansicht zufolge, für idg. *diwos-suHmus 'Solnn des Zeus' halten; diese Deutung wird von JANDA zurecht zurückgewiesen, denn sie verletzt die Lautgesetze: *diwos-sullnus hätte sich weder zu Dionysos noch zu einer anderen der bezeugten Varianten dieses Theonyms (LSI, S. 433, s.v. $\Delta$ (óvuoọ) entwickelt.

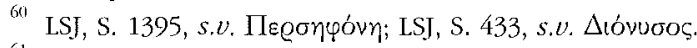

61 F. GSCHNiTzer macht mich darauf aufmerksam, dass die Varianten auf -pov- bzw. $-\varphi \alpha \tau \tau-$, die von Persephone existieren, nach altem idg. Ablaut auszusehen scheinen und dass hier vielleicht ein besonders altes und deswegen verdunkeltes, aber sehr wohl idg. Hinterglied vorliegt, und die Verdunkelung auch auf das Tabu, den Namen der Todesgöttin auszusprechen, 
Angesichts der Wichtigkeit der göttlichen Epiphanie in der minoischen Religion des 2. Jt. könnten viertens vielleicht auch Auffälligkeiten im Kultgeschehen wie die charakteristische Epiphanie der Gottheit (zweifelsfrei der Höhepunkt der eleusinischen Mysterien, vgl, unten, S. 30) dem nicht-idg. Bereich zuzuordnen sein. ${ }^{6}$

Umgekehrt gibt es nach wie vor keine indogermanische Etymologie der $D \bar{a}-m \bar{a} t \bar{e} \cdot / D \bar{e}-m \bar{e} t \bar{e} r$; die in bezug auf $D \bar{a}-1 D \bar{e}$ - sowohl lautlich als auch bedeutungsmässig befriedigend wäre. ${ }^{63}$ Vor diesem Hintergrund wird es unwahrscheinlich, dass die Ursprünge des Demeterkults im indogermanischen Bereich anzusiedeln sind. ${ }^{61}$

Es kann aber kaum bezweifelt werden, dass dasselbe ungedeutete Element $d \bar{a} / d \bar{e}$ auch im Götternamen Poseidon (<Poseidāōn) auftritt, ${ }^{65}$ und dass Poseidabei als der alte Vokativ von $\pi$ óoı 'Herr', 'Gatte', Poseid̄̄ōn also als 'Gatte der $D \bar{a}-$ ' zu verstehen ist. ${ }^{6 \sigma}$ Poseidon und Demeter treten im Mythos der abgelegenen Landschaft Arkadien als Paar auf (Paus., VIII, 25, 5 und 42, 1), und man wusste dort von den beiden Gottheiten besonders Eigenartiges, allem Anschein nach sehr Altertümliches, zu berichten, ${ }^{67}$ wobei deren wiederholte Nähe bzw. Identität mit einem Pferd besonders auffällig ist. Auch die arkadischen Demeter- und Poseidonkulte wirken altertümlich, ${ }^{6}$ was zur Vermutung führt, dass diese beiden Gottheiten im Herzen der Peloponnes, dem einstigen Rückzugsgebiet der Mykener, gewisse aus der mykenischen Zeit ererbte Züge haben bewahren können. ${ }^{69}$

Im Gegensatz zu Demeter ist Poseidon auf den mykenischen Tontäfelchen bezeugt; zumindest in Pylos muss er der wichtigste Gott gewesen sein. ${ }^{70}$ Die Hauptgöttin von Pylos war dagegen, wie ich kürzlich gezeigt zu haben

zurïckzuführen sein könnte. Im Falle von Dionysos dagegen mag der Gen. des idg. Zeus zugrundeliegen; angesichts des etymologisch unverständlichen Hintergliedes scheint es mir aber auch möglich, dass Dionysos auf Grund einer volksetymologischen Umdeutung eines vorgriechischen Theonyms zum Sohn des Zeus wurde (wohl in vormyk. Zeiten, da Dionysos und Zeus gemeinsam auf einem myk. Täfelchen von Kydonia bezeugt sind, vgl. z.B. J.-P. OLIVIER, BCH 117 [1993], S. 19-33).

62 Zu einem möglichen nicht-idg. Einfluss ägyptischer Herkunft im eleusinischen Kult unten, Anm. 101.

63 Vgl. oben, Anm. 53 (Sekundärliteratur zur Etymologie).

${ }^{6}$ Dazu passt gut, dass der Name Damater in einer Inschrift aufzutauchen scheint, die in einem minoischen Gipfelheiligtum in Kythera gefunden wurde und mit Linear A-Schriftzeichen verfasst ist, vgl. Y. DuHOux, Minos 29f. (1994-95), S. 289-294.

${ }^{65}$ So schon P. KRETSCHMer, l.c. (Anm. 48); zuletzt M. JANDA (2000), S. 256.

${ }^{66}$ F. GsChNITZER $(1962)=i d .(2001)$, S. 294-299.

67 Altertümlich wirken z.B. PAUs., VIII, $8,1-2 ; 10,1-4 ; 14,5 ; 15,1-4 ; 25,2-10 ; 31$, 1f. und $7 f$; 37,$10 ; 42,1-7$ u.a.

${ }^{63} \mathrm{Zu}$ diesen arkadischen Mythen und Kulten M. JOST (1985), S. 279-355; M.P. NILSSON (1967), S. 447f., S. 477-481; zu Poseidons Pferdecharakter auch J. BREMMER, DNP 10 (2001), Sp. 201f., s. $v$. Poseidon. $\mathrm{Zu}$ den Poseidonkulten der Peloponnes allgemein zuletzt J. Mrtoropoulos, Heiligiümer und Kulte des Poseidon auf der Peloponnes, Liège, 2003 (Kernos, suppl. 13).

(1) F. GRAF, DNP 3 (1997), Sp. 424, s.t. Demeter; M. JOST (1985), S. 351-355.

7) W. Buriert (1977), S. 84 = id. (1985), S. 44; M. GÉrard-Rousseau (1968), S. 181-185. 
glaube, Potnia, ${ }^{71}$ sprachlich gesehen die alte Femininbildung von $\pi$ ó $\iota \varsigma$ (jünger im ion. Dialekt $\pi$ óøı) , also ein deutliches indogermanisches Relikt: Neben dem 'Herrn' der $D \bar{a}$ gab es im 2. Jt. die 'Herrin', mit der Poseidon auch nach dem mykenischen Textmaterial zu schliessen liiert gewesen zu sein scheint. ${ }^{72}$ In nachmykenischen Zeiten fristet Potnia zwar nur noch ein schattenhaftes Dasein, wo Potnia im 1. Jt. aber noch als Theonym belegt ist, handelt es sich im Singular um die Bezeichnung Demeters oder ihrer Tochter, ${ }^{73}$ und im Plural um diejenige dieser beiden Göttinnen. Der Schluss drängt sich auf, dass im 2 . Jt. eine dem vorgriechischen Substrat zuzuordnende Gottheit $D \bar{a}$ ( $-m \bar{a} t \bar{e} r)$ von der indogermanischen Bevölkerungsschicht mit dem altererbten Begriff Potnia benannt wurde, ${ }^{74}$ umso mehr, als in den mykenischen Texten von einer si-topo-ti-ni-ja ('Herrin des Getreides') und einer po-ti-ni-ja i-qe-ja (Potnia bippeia, $c f$. den genannten Poseidon i $\pi \pi \iota \varsigma$ ) die Rede ist, die einerseits auf Demeter als Getreide-Göttin und andererseits auf ihre Verknüpfung mit Pferden in Arkadien verweisen. Es kann sein, dass idg. Potis und idg. Potnia als so benannte Gottheiten ${ }^{75}$ im griechischen Raum auf vorgriechische Gottheiten des Substrats gestossen sind, mit denen sie eine Symbiose eingegangen sind. Ebenso gut denkbar ist, dass diese beiden Begriffe als idg. Bezeichnungen für zwei alteingesessene Gottheiten des Substrats zu verstehen sind, die die Einwanderer irgendwann nach 2000 übernommen hätten. Potnia bzw. Poteimögen dabei als Lehnübersetzungen zu verstehen sein. Wie dem auch sei, die einheimische Bevölkerung muss eine grosse Göttin $D \bar{a}(-)$ gekannt haben, die mit Potnia prinzipiell identisch war. Dabei dürfte Potnia auch spezifische, bei

71 C. TRÜMPY (2001): Der auf den mykenischen Täfelchen häufig wiederkehrende und eindeutig dem kultischen Bereich angehörende Begriff po-ti-ni-ja /potnia ist, den Wortbildungsregeln entsprechend, zweifelsfrei als Substantiv zu verstehen ('die Mächtige', 'die Herrin'), das nach einer textimmanenten Interpretation des mykenischen Materials ebensogut für verschiedene weibliche Gottheiten wie für eine einzige, unter verschiedenen Aspekten verehrte, in Gebrauch gewesen sein kann. Der Gebrauch von $\pi \delta ́ \tau v \iota \alpha$ im 1. Jt. lässt sich aber nur erklären, wenn wir von der Variante "Polnia $=$ eine Gottheit" ausgehen und myk. Polnia folglich als eine Art Theonym deuten, ähnlich wie frz. Vierge oder engl. Lady.

72 Auffällig ist erstens die Serie Fr von Pylos, in der Poseidon als erster Gabenempfänger erwähnt wird, gefolgt von mehreren Zeugnissen für Potnia als Gabenempfängerin. Zweitens finden sich in einer anderen Liste von Opfergaben aus Pylos, auf dem Täfelchen Fn 187, nebeneinander die Einträge u-po-jo po-li-ni-ja ('Potnia von U.' [Toponym]), po-si-da-i-je-li-si (Dat. Pl.: 'Poseidonpriestern') und po-si-da-i-jo-de ('in das Heiligtum des Poseidon'). Drittens steht auf der Tafel Tn 316 von Pylos Potnia zu Beginn der Empfängerliste, während zuoberst auf dex Rückseite der Eintrag po-si-da-i-jo erscheint. Vgl. C. TRÜMPY (2001), S. 419f.

${ }^{73}$ Cf. unten, S. 30.

74 J. Chadwck, der als erster eine Studie über Potnia vorgelegt hat (Minos 5 [1957], S. 117129), glaubt, dass Potnia die griechische Bezeichnung der grossen minoischen Fruchtbarkeitsgöttin war, die man erst später unter dem Namen Demeter verehrt hätte.

${ }^{75}$ Im Sanskrit ist palmi (<* ${ }^{*} \circ m_{2} H_{2}$ wie myk. Potnia) die Bezeichnung für die Gattin eines Gottes (z.B. Rigveda I, 103, 7, Plural), was angesichts des ebenfalls religiösen Charakters von myk. Polnia die Vermutung nahelegt, alle beide könnten auf ein gemeinsames altes, aus idg. Zeit stammendes Theonym * potniH $I_{2}$ zurückgehen. Daher wurde im oben (Anm. 71) zitierten Artikel über Polnia der Vorschlag gemacht, Polnia und ihr männliches Gegenstück Posei(don) als letztendlich idg. Gottheiten zu deuten. 
$D \bar{a}(-m \bar{a} t \bar{t} r)$ nicht vorhandene, mit der Palastkultur in Zusammenhang stehende Charakteristika entwickelt haben. Dafür spricht das weitgehende Verschwinden des Theonyms Potnia im 1. Jt., das zur Vermutung Anlass gibt, Potnia sei als Palastgöttin des Königshauses nach dem Zusammenbruch der mykenischen Paläste um 1200 zusammen mit der Palastkultur untergegangen so wie der wanax und die ganze königliche Verwaltung, ${ }^{76}$ während das Wissen um die ehemalige Fastidentität von Potnia und Demeter in den überlebenden Bauernkulturen das vereinzelte spätere Auftauchen des Theonyms Potnia bzw. Potnial für Demeter und ihre Tochter erklären würde.

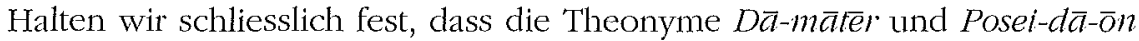
alle beide eine Mischung aus indogermanischem und einheimischem Sprachgut darstellen, wobei der idg. Teil einmal voran- und einmal nachgestellt ist. ${ }^{77}$ Die Vereinigung je eines einheimischen und eines idg. Wortbestandteils in den Namen zweier wichtiger Gottheiten spiegelt besonders schön die Verschmelzung indogermanischer Vorstellungen mit denen des Substrats.

Einer derartigen Sichtweise scheinen sich die Informationen auf den neuen Täfelchen aus Theben ${ }^{78}$ zu widersetzen, wo nach den Herausgebern V.L. Aravantinos, L. Godart und A. Sacconi eine Göttertrias zu identifizieren sei, die die Gottheiten ma-ka ( $M \bar{a} G \bar{a}$ 'Mutter Erde'), o-po-re-i (Opōres 'Früchtebringer', Epitheton von Zeus) und ko-wa (Korwā 'Mädchen') enthalte und hinter der sich die drei Gottheiten Demeter, Zeus und Persephone verbergen sollen (TH Fq 126 et al.). ${ }^{79}$ Wenn diese Hypothese zuträfe, hätten wir hier die frühesten Zeugnisse für den später auf Eleusis beschränkten Kult der Demeter

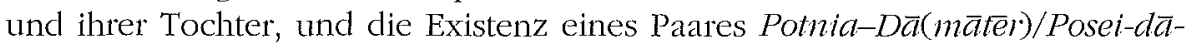
$\bar{o} n$ in mykenischer Zeit wäre durch die Bezeichnung der Göttin als $M \bar{a} G \bar{a}$ und durch deren Verbindung mit Zeus in Frage gestellt. Entscheidende Argumente sprechen aber gegen die Hypothese von V.L. Aravantinos et al.:.

76 Vgl. C. TRÜNPY (2001), S. 417.

${ }^{77}$ Dă-mätêr: "Zusammentückung" (mătê: ursprüngl. Apposition); Poseidon: mit -ōn erweitertes Kompositum.

78 S. oben, Anm. 2.

79 V.L. ARAVANTINOs et al. (2001), S. 188-194; dementsprechend schon fiz̈her; z.B. L. GODART; A. SACCONI (2000), S. 19 .

8! Vgl. auch T.G. PALAIMA, Minos 35f. (2000-2001), S. 475-486, und AJA 107 (2003), S. 113-115 (Rez. von V.L. ARAVANTINOS et al. [2001]); In diesen vorbildlichen Besprechungen weist auch Palaima mit guten Gründen die Deutung von ARAvANTINOS ef al. zurück und äussert sich angesichts des Fehlens eindeutiger Theonyme in neuen thebanischen Material zurecht skeptisch gegenüber einer Eingliederung der betreffenden Täfelchen in einen religiösen Kontext. Dementsprechend auch in seinem handout für das Internalional Symposium on Thebes, 16-17 November 2002, S. 7: "Most important, however, is that I advise that we all read the tablets in the $\mathrm{Fq}$ series as modern proofreaders read texts, that is backwards. Proofreaders do this to avoid assumptions that would cause them to overlook mistakes. This procedure will help us also. If we start with the totals and read backwards, we will see that there is no compelling reason to interpret any of the entries in these long texts in a religious sense. The possibility may still exist that $m a-k a$, which is the first entry on all 15 or 18 of these routine and repetitive texts, could be the sole exception and be interpreted as Ma Ga. But both in these texts and in its one other occurrence Knossos tablet F 51, I think it is more reasonable to interpret ma-ka as one of the 
Erstens mag zwar ma-ka in Theben eine grosse Göttin gewesen sein, vielleicht sogar die Hauptgottheit des bronzezeitlichen Theben, die genau wie Demeter mit Gerste assoziiert wurde. Aus verschiedenen Gründen ist aber die Annahme, ma-ka sei als $M \bar{a} G \bar{a}$ mit Demeter zu identifizieren und in den Kontext der eleusinischen Mysterien einzuordnen, zurückzuweisen. Das Argument von Aravantinos et al. (S. 190ff.), die Deutung $M \bar{a} G \bar{a}$ 'Mutter Erde' $=$ Demeter werde durch vereinzelte literarische Zeugnisse gestützt, ist nicht stichhaltig. Diese Zeugnisse deuten zwar z.T. das (schon im Altertum nicht mehr verstandene) sprachliche Element $D \bar{a}(-) / D \bar{e}(-)$ mit einer Gottheit $G \bar{a} / G \bar{e}$ - wegen des ähnlichen Klanges und des auf den ersten Blick an eine Erdgöttin erinnernden Charakters von Demeter. ${ }^{81}$ Demeter war aber in keiner Art und Weise eine „Mutter Erde“. Sie war zuständig für die Erhaltung der Fruchtbarkeit im weitesten Sinn und spendete Trost in Hinblick auf das menschliche Schicksal nach dem Tod, wie wir den Quellen für die Thesmophorien und die eleusinischen Mysterien entnehmen können. Vor allem aber heisst Dāmăter bzw. Demeter, den unzähligen Belegstellen für diesen Namen zum Trotz, immer so, mit D- im Anlaut, niemals **Gämäter bzw. **Ge mēter: Dabei ist entscheidend, dass Poseidon, myk. Poset-dă-ön (zahlreiche myk. Belege, niemals **Poseiḡ̄on) die Namensform $D \bar{a}$ - bereits für die mykenische Zeit belegt. Ferner passt nach unseren Ausführungen über die (Fast-)Identität von myk. Potnia mit Demeter gut ins Bild, dass Potnia auf den mykenischen Täfelchen von Theben direkt bezeugt ist. ${ }^{82}$ Aus diesen Beobachtungen folgt aber, dass theb. ma-ka - selbst wenn sie eine $M \bar{a} G \bar{a}$ 'Mutter Erde' sein sollte - mit Demeter nichts zu tun haben kann. Angesichts der mykenischen Orthographie kann ma-ka aber ebenso gut /Marga/, /Manga//, /Markā/, /Mankä/, /Mankba/a/ etc. gelesen werden. Wenn wir es überhaupt mit einer Gottheit zu tun haben, ist es angesichts der Tatsache, dass von den zahlreichen im mykenischen Textmaterial belegten Theonymen im 1. Jt. ca. die Hälfte verschollen bleibt, sehr wahrscheinlich, dass sich hinter ma-ka in Wirklichkeit eine später ganz unbekannte alte Lokalgottheit verbirgt. ${ }^{83}$

Zweitens ist die genannte Deutung von o-po-re-i, das Aravantinos et al. mit Zeus - im 1. Jt. Vater der Persephone - in Zusammenhang bringen, alles

frequent Mycenaean nomina actionis: $m a-k a=m a g \bar{a}=* \mu \alpha y \dot{n}$ 'the action of kneading barley into ceremonial cakes'. This term occurs as a leading entry in the Fq texts because the first allocation of a much larger quantity of barley is made for processing into ceremonial barley cakes for the kind of ritual banquet that Homer would clesignate as a $\delta \alpha i c_{\text {. }}$

${ }^{81}$ Dabei ist die vielzitierte Stelle Aischr't., Supp., 890f., insofern nicht relevant, als die "Erde" an eben dieser Stelle als Mutter (Grossmutter?), und nicht als Gattin von Zeus erscheint ( $\tilde{\omega} \Pi \tilde{\alpha}$,

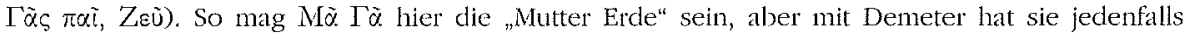
nichts zu tun.

${ }^{82}$ TH Of 36: po-ti-ni-ja wo-ko-de potniās woikonde 'in das Haus der Potnia' bzw. 'in den Tempel der Polnia'.

83. Die Herausgeber der mykenischen Täfelchen von Theben lesen nun einen Beleg aus IKnossos (F 51), der vor dem Bekanntwerden dieser Täfelchen für unklar galt, ebenfalls als ma-ka, vgl. V.L. Aravantinos et al. (2001), S. 188. Zweifel sind angebracht. Aber auch wenn ma-ka eine überregional bekannte Gottheit gewesen sein sollte, ändert dies nichts an der Unmöglichkeit, maka mit Demeter in Verbindung bringen zu können. 
andere als gesichert. Es handle sich um den Dativ des Substantivs *opōres, Epitheton von Zeus in der Bedeutung 'Früchtebringer' o.ä. (zu ó $\pi \omega ́ \omega \alpha$ 'Spätsommer'). ${ }^{8 \dagger}$ Auszugehen hätten wir dann von einem alten $s$-Stamm. Als Stütze dient die Beobachtung, dass eine böotische Inschrift (IG VII, nr. 2733,

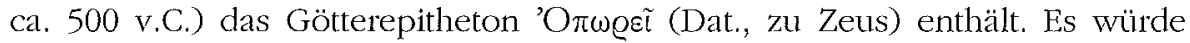

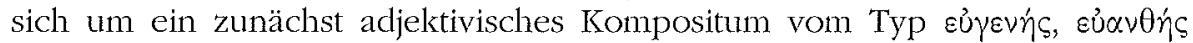
usw. ${ }^{85}$ handeln. Sehr unschön bei dieser Erlklärung ist aber erstens die Tatsache, dass ỏ $\pi \omega \varrho \alpha$ kein $s$-Stamm ist und dass es keinerlei Hinweise gibt, dass dieses Substantiv je als $s$-Stamm existiert hat; denn die grosse Mehrheit der adjektivischen $s$-Stämme enthält erwartungsgemäss einen nominalen $s$ Stamm. ${ }^{86}$ Ebenso unschön ist es zweitens, ausgerechnet in der mykenischen Zeit von einer morphologischen Neuerung ausgehen zu müssen, umso mehr, wenn o-po-re-i dem Kultvokabular angehört, das tendenziell zu altertümlichen Wortformen neigt. Umgekehrt erwarten wir ein nomen agentis zu ó $\pi \omega \varrho \alpha$ am

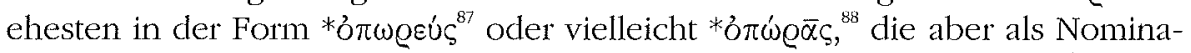
tive von o-po-re-i selbstverständlich alle beide ausser Betracht fallen. ${ }^{89}$ Demnach müssen wir damit rechnen, dass die Aehnlichkeit von o-po-re-i bzw. böot. 'O schreiben ist.

T.G. Palaima ${ }^{90}$ (und unabhängig von ihm auch F. Gschnitzer in einer mündlichen Besprechung) schlagen vor, o-po-re-i mit ỏ $\pi(=\varepsilon \dot{\varepsilon} \pi)$ und ógos 'Berg' zu verbinden; o-po-re-i würde damit etymologisch gesehen 'derjenige auf dem Berg' bedeuten. Diese lautlich und morphologisch einwandfreie Deutung ist als Name eines sterblichen Mannes überzeugend; mit einem „human recipient" rechnet denn auch Palaima. Für eine Gottheit ist sie alles andere als naheliegend und lässt sich auf jeden Fall nicht weiter stützen.

Vielleicht verbirgt sich auch hinter dieser Bezeichnung ein nicht etymologisierbarer Name, der im übrigen in der mykenischen Zeit auch unter der Annahme, es handle sich um eine göttliche Gestalt, noch keineswegs ein Beiname von Zeus gewesen zu sein braucht, sondern durchaus eine unabhän-

${ }^{84}$ Zu Recht bemerken ARAVANTINOS et al., dass nicht von einem Nom. *Oporeus ausgegangen werden dürfe, da *Oporeus auf dem Täfelchen in der Form *o-po-re-we erschiene. (Dieser Irrtum. bei A. Schachter, „Greek Deities: Local and Panhellenic Identities“, in P. FlEnSTED-JenSEN (ed.), Furtber Studies in the Ancient Greek Polis, Stuttgart, 2000, S. 14.)

${ }^{85}$ Zu diesem Typ z.B. P. Chantraine (1933), S. 424-429.

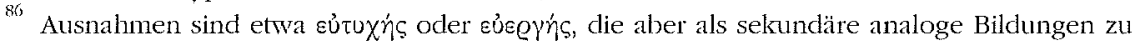
verstehen sind.

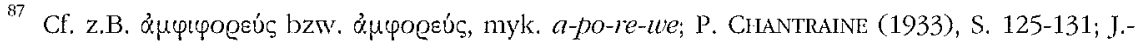
L. Pfrpillou (1973), Beispiele auf den S. 81-161; A. Lethart (1994), S. 240-268.

88 P. Chantraine (1933) S. 27; A. LeukarT (1994), S. 204-229.

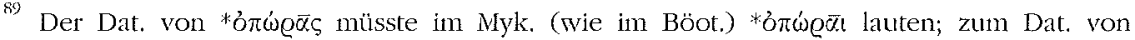
*ỏnwgeús oben, Anm. 84.

90 Minos 35f. (2000-2001), S. 479; AJA 107 (2003), S. 115; Handout für das International Symposium on Thebes, 16-17 November 2002, S. 8. 
gige Gottheit gewesen sein kann. Wie dem auch sei, auf jeden Fall spricht nichts dafür, dass o-po-re-i als Teil einer Göttertrias zu verstehen wäre."

Was ko-wa betrifft, das mehrfach nach ma-ka erscheint und zweifellos als /korwāi/, Dat., 'dem Mädchen' zu verstehen ist, gehen die Herausgeber der neuen Täfelchen von Theben davon aus, dass "pour la première fois, les tablettes en linéaire B nous restituent le théonyme Kógn = Korè. Korè, selon la tradition d'Éleusis, est fille de Déméter et de Zeus" (S. 190). Nach unseren Ausführungen über $m a-k a$ und $o-p o-r e-i$ ist dies aber ganz ausgeschlossen. Mit ko-wa muss hier ein anderes 'Mädchen' gemeint sein, das einen göttlichen Status gehabt haben mag: Im 1. Jt. wurde xó@n im göttlichen Bereich durchaus nicht nur von Demeters Tochter gebraucht, sondern auch etwa von den Eumeniden u.a. (s. z.B. LSJ, S. 980f., s.v. xó@ך).

In unserem Zusammenhang ergibt sich damit, dass die Bezeichnung ma$k a$ in keiner Art und Weise die Existenz des Theonyms $D a \bar{a}$ (mäter) bereits im 2. Jt. in Frage zu stellen vermag.

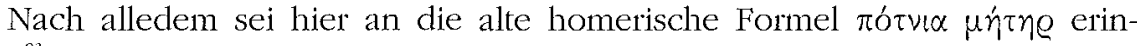
nert. ${ }^{93}$ Sie ist in der Ilias $21 \mathrm{mal}$ und in der Odyssee 13 mal vertreten, immer am Versende, das bekanntlich besonders deutlich zur Formelhaftigkeit neigt, und bezeichnet ganz unterschiedliche Mütter, in der llias besonders oft ( 8 mal) Thetis, die dort von den Müttern die wichtigste Rolle spielt. Nach

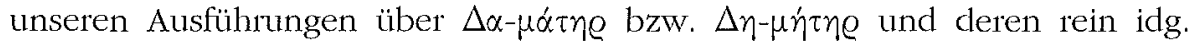
Bezeichnung Potnia ist es vielleicht nicht zu gewagt, die Frage zu stellen, ob wir die Ursprünge dieser Formel nicht in die Zeit vor der Katastrophe von 1200 zurückdatieren dürfen, als die grosse Göttin Potnia unter dieser

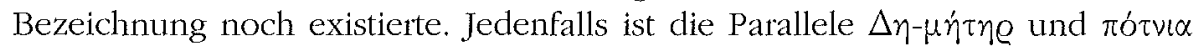

91 Ebensowenig akzeptabel ist im Falle des Täfelchens TH Fq 130 (... o-je-ke-te-to ma-ka ...) die von V.L. ARAvANTINOs et al. (2001), S. 195f., vorgeschlagene Deutung von o-je-ke (te-to sei eine Verbform von $\tau(\theta \eta \mu)$ ) „Dans ce mot (scil. o-je-ke) on peut voir le substantif *óetyŕs avec le jod utilisé comme phonème de transition (glide) entre $o$ et $e$. Un tel terme appartiendrait au groupe des substantifs en - $\eta$ s qui font partie de la famille des noms avec suffixe $-s$, la même famille à laquelle appartiennent les neutres en -os et les adjectifs en - $\rangle_{\zeta},-\varepsilon \varsigma . "$ Die gleiche irtümliche Deutung findet sich bei L. GODART, A. SACCONI (2000), S. 21f. Das Problem besteht darin, dass es im Griechischen keine s-stämmigen Substantive mit einem Nom, auf -ës gibt. (Zitiert wird auf der S. 195, Anm. 77, bzw. auf der S. 21, Anm. 7, E. SCHWYZER (1939), S. 511f. und 578580; dort ist auf S. 579 die Rede von homerischen mask. und fem. mit einem Nominativ auf $-\eta \subseteq$ unter Verweis auf lat. Ceres und Venus, Veneris; dies düifte ARAvANTINOS, GODART und SACCONI zu ihrer Interpretation gebracht haben; was für hom. Beispiele SCHWYZER meint, ist unklar; E. Risch (1974), und P. Chantraine (1939) wissen davon jedenfalls nichts; und lat. Ceres wie Venus werden von A. ERNOUT-A. MEILLE, Dictionnaire étymologique de la langue latine, Paris, $1959^{\prime}$, s.v., für umgedeutete alte Neutra gehalten.) Somit wird die Schlussfolgerung von ARAVANTINOS, GODART und SACCONI hinfällig, wonach *óely j́s 'ouverture' oder 'révélation' heisse; und es kann keine Rede davon sein, dass wir mit 'ouverture (sous-entendu de la fête ou du rite)' übersetzen könnten bzw. dass wir Grund hätten zu „penser à quelque chose de similaire à la révélation des Hiera à l'occasion de l'initiation aux cérémonies des mystères d'Éleusis" (ARAVANTINOS et al. [2001], S. 196).

22 Ein weiteres Argument zugunsten dessen damaliger Existenz unten, S. 30f. (Deo im Demeterhymnus).

93 C. TRÜMPY (2001), S. 416. 
$\mu \eta \dot{i n} \varrho$ nach der Entdeckung von deren (weitgehenden) Identität sehr auffällig.

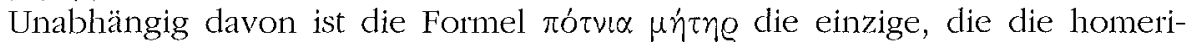
sche Umdeutung des alten, unabhängigen Substantivs Potnia zu einer adjektivisch gebrauchten Apposition" einleuchtend zu erklären vermag," was die

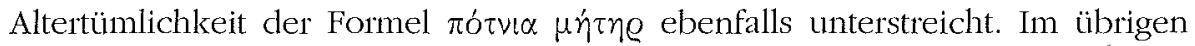
steht die Apposition im Griechischen meist nach ihrem Bezugswort," was offenbar altererbtem Sprachgebrauch entspricht. ${ }^{y 7}$ Auch dies spricht dafür,

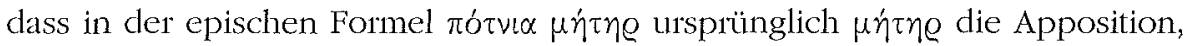
$\pi o ́ \tau v l \alpha$ dagegen das Theonym war. Am einfachsten scheint mir anzunehmen, dass in mykenischer Zeit das Syntagma Potnia mēter in Gebrauch war, und dass sich dieses nach 1200 , als Potnia als eigenständige Gottheit verschwunden war, umgedeutet zu 'die Herrin', 'die Mutter' bzw. 'die hehre Mutter' o.ä., im Heldenepos hat etablieren können. Die Heldenepik tichtete sich zunächst an den Adel, der dadurch an seine glorreiche Vergangenheit erinnert wurde. ${ }^{98}$ In diesem Rahmen passt ein Weiterleben der einst mit dem mykenischen Palast verbundenen Potnia besonders gut. Demgegenüber hat sich Demeter nur einen marginalen Zutritt zum Epos verschaffen können, was zweifellos zurecht mit ihrem im Volk verankerten Wesen erklärt wird, von dem sich der Adel zu distanzieren suchte."

\section{Gedanken zu den eleusinischen Mysterien, zu Brimo, Deo und einer alten Hymnendichtung}

Unter dem Begriff „Mysterien“ ( $\mu \nu \sigma \tau \hat{\jmath} \varrho\llcorner\alpha)$ verstehen wir eine spezielle Art von Kulten innerhalb der griechischen und der römischen Welt. ${ }^{100}$ Allen Mysterien gemeinsam war ein Aufnahmeverfahren durch Initiation sowie Geheimhaltung der wesentlichen initiatorischen Elemente, wodurch sich die Eingeweihten als besondere Gruppe verstanden. Charakteristischerweise enthielten die Mysterien Versprechungen auf Verbesserungen der Verhältnisse im Diesseits sowie Hoffnungen auf ein gutes Los im Jenseits. ${ }^{101}$ Die Einweihung war der freien Entscheidung jedes einzelnen überlassen und die

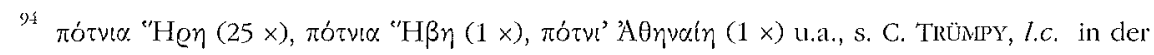
vorhergehenden Anm.

25 Vgl. C. TRÜMPY (2001), S. 418.

G. E. E. SCHWYZER (1950), S. 615 und S. 618.

97 Vg1. lat. Juppiter <*Djou pater; skr. Dyaub pitar:

98 E.g. A. LESIKY (1971), S. 29-34.

29) Auch Dionysos wird, offenbar aus dem gleichen Grund wie Demeter, in der Heldenepik vermieden. Vgl, auch E. Rusch (1981), S. 346-353, wo er nachweist, dass typisch umgangssprachliche Wendungen in der epischen Kunstsprache fehlen.

${ }^{100}$ W. BURKERT (1977), S. 413-451 =id. $(1985)$, s. 276-304; W. BURKERT $(1987)=i d .(1990)$.

${ }^{101}$ Vermittlung von Trost für das Jenseits ist innerhalb des griechischen Kultlebens aussergewöhnlich und könnte ursprünglich von ägyptischen Vorstellungen beeinflusst sein. Die ägyptische Möglichkeit der Erlösung aus der Todeswelt hätte eine Ausstrahlung einerseits in die griechischen Mysterien und andererseits ins Christentum erfahren: e.g. J. ASSMANN, in J. ASSMANNM. BOMmas (eds), Alegpptiscbe Myslerien, München, 2002, s. 75. 
Teilnahme stand Männern und Frauen, ja sogar Sklaven und Fremden offen. Im übrigen waren jedoch die verschiedenen Mysterienkulte voneinander verschieden. Die Mysterien von Eleusis ${ }^{112}$ nahmen einen besonderen Rang ein und entfalteten eine einmalige Breitenwirkung: Die meisten Athener waren eingeweiht, aber auch unzählige Menschen aus ganz Griechenland und später aus dem gesamten römischen Reich wurden durch diesen Kult angelockt, bis zu dessen Verbot durch Theodosius und der Zerstörung des Heiligtums in Eleusis durch die Goten um 400 n.C. Das Fest fand jeweils im Herbstmonat

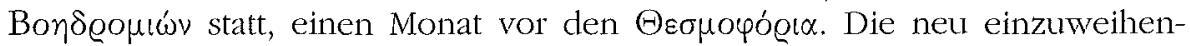

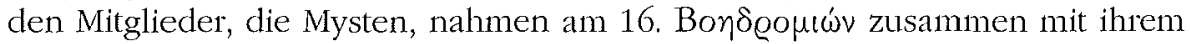

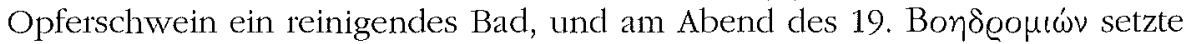
sich dann der Zug aller Teilnehmer - nebst den Mysten auch die sogenannten

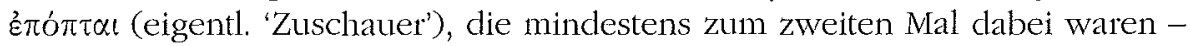
nach dem ca. $25 \mathrm{~km}$ entfernten Eleusis in Bewegung. Die eigentliche, nächtliche Feier fand im sogenannten Telesterion, einem riesigen quadratischen Bau ( $52 \mathrm{~m} \times 52 \mathrm{~m}$ ), statt. Von den Geschehnissen im Telesterion ist nur wenig bekannt. Die Teilnehmer waren dabei offenbar von völligem Dunkel umgeben, während die Priester im Fackelschein agierten und Angsterregendes darstellten. Schliesslich erschien, als Beglückung, Kore aus der Unterwelt, und der oberste Priester, der Hierophant, zeigte eine abgeschnittene Aehre und

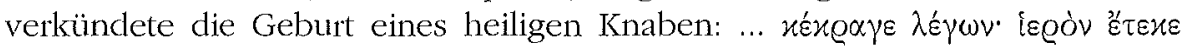

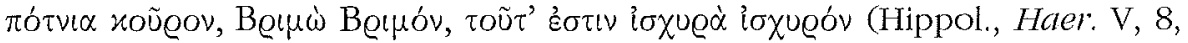
40). ${ }^{103}$

Diese Verkündigung ist nun aber in mehrfacher Hinsicht bemerkenswert.

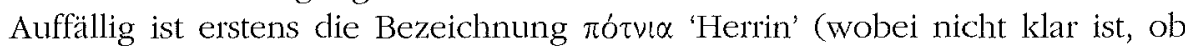
mit $\pi$ ó vil $\alpha$ Demeter oder ihre Tochter gemeint ist). Denn abgesehen von

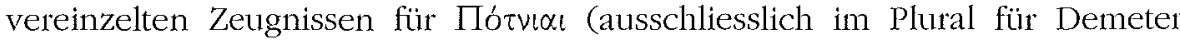
und Kore) ist $\pi$ ó $\tau$ la in der Umgangssprache des 1. Jt. nicht belegt ('Herrin'

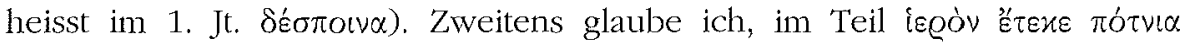

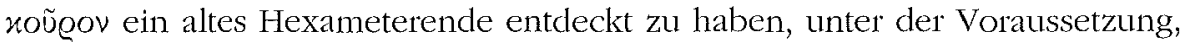
dass das Augment des Verbs sekundär hineingeraten ist. Da das Augment in der homerischen Metrik fakultativ ist, und ferner die Wortstellung von íeóv und roũeov (Hyperbaton) ohnehin für eine dichtersprachliche Wendung spricht, scheint es mir sehr naheliegend anzunehmen, dass hier ein Bestandteil aus einer traditionellen Dichtung vorliegt. Dafür spricht auch die Form

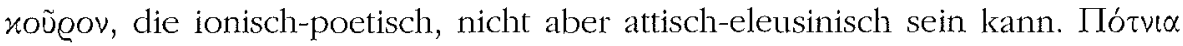
ist aber bei Homer fast nur als de facto adjektivische Apposition (v.a. in den

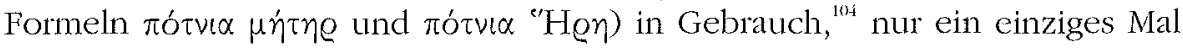

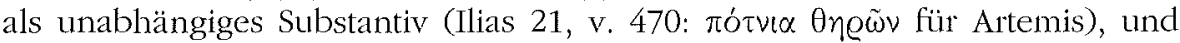
zudem kommt Demeter im Epos praktisch nicht vor. ${ }^{105}$ Demnach fehlt ein

102 W. BUIRFFrT (1977), S. 426-432 = id. (1985), S. 285-290.

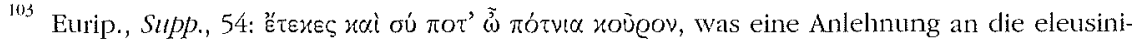
sche Formel darstellen muss, vgl. N.J. RiCHARDSON (1974), S. 26f.

101 Vgl. oben, Anm. 94.

1015 S.O. S. 30. 
Tradition vermutet werden darf. Zu dieser Annahme passt insbesondere auch

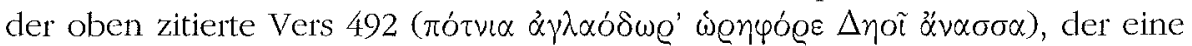
für einen Hymnus typische Häufung von Kultepitheta enthält ${ }^{117}$ und hochtraditionell wirkt. Denn $\pi$ ó $v \dot{ } \alpha$ ist am einfachsten wie in der eleusinischen Verkündigung als unabhängiges Kultepitheton zu verstehen und folglich mit besonders hoher Wahrscheinlichkeit einer nicht-homerischen Hymnentradition zuzuordnen; zudem sieht auch das altertümliche $\alpha^{\prime} v \alpha \sigma \sigma \alpha$ nicht nach einer Entlehnung aus dem Epos aus. ${ }^{118}$

Alles in allem werden wir festhalten, dass zwar ein stringenter Beweis dafür, dass $\pi$ ótvı $\alpha$ und $\Delta \eta \hat{\omega}$ im Demeterhymnus auf eine vorliterarische, mündlich tradierte hexametrische Hymnentradition zurückgehen, nicht möglich ist, dass die verschiedenen vorgebrachten Indizien aber dafür zu sprechen scheinen, dass letztlich uralte, in den Dark Ages (oder sogar in der Bronzezeit?) fussende Kultdichtung zugrunde liegt. Die bronzezeitliche

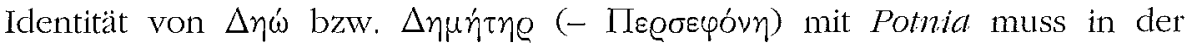
Kultdichtung lebendig geblieben sein, womit sich auch die im 1. Jt. vereinzelt auftretende Bezeichnung Пótvı $\alpha$ für Demeter und ihre Tochter bestens erklärt. Da diese Kultdichtung für die zu ehrende Gottheit verschiedene Bezeichnungen verwendet hat, mag auch die bei Homer so verbreitete

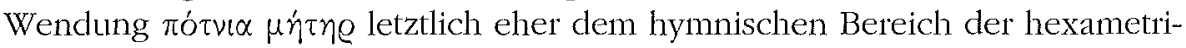

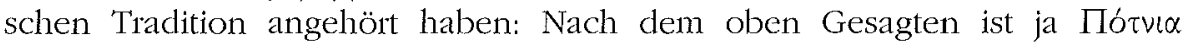

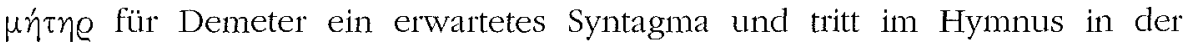
erwarteten Bedeutung auf, während der homerische Gebrauch, wie oben beschrieben, sekundär ist.

\section{Das Anaktoron}

Im Innern des eleusinischen Weihehauses, des sogen. Telesterions, lag ein länglicher, quaderförmiger Steinbau mit einer Türe am Ende der einen Längswand, der sich trotz verschiedener Bauphasen des Telesterions immer an derselben Stelle befand: das sogenannte $\alpha \dot{\alpha} \alpha \dot{x}$ to@ov. ${ }^{119}$ Diese Bezeichnung war allem Anschein nach ursprünglich im eleusinischen Demeterkult zu Hause $^{120}$ und breitete sich in der allgemeinen Bedeutung 'Heiligtum' auch anderweitig aus, besonders in der Kaiserzeit. In der byzantinischen Zeit und

117 Zur Gewohnheit, die Gottheit mit unterschiedlichen Namen anzurufen, W.D. FurLEY, DNP 5 (1998), Sp. 789, s.v. Hymnos, Hymnus; id., Greek Hymns I, Tübingen 2001, s. 52-56.

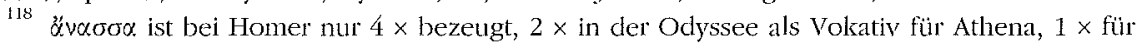
Nausikaa, die dabei von Odysseus für Athena gehalten wird, und $1 \times$ in der Ilias (XIV, 326: ...

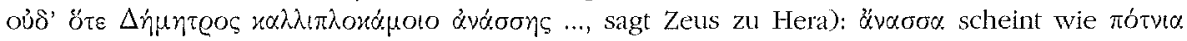
am ehesten aus einer alten Kultsprache zu stammen.

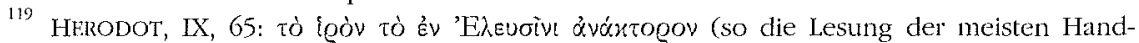
schriften, neben der Lesung àvaxtógtov); weitere Stellen bei L. Deuiner (1932), S. 87-90.

12i) Dort ist sie seit Herodot gut bezeugt, während die Übertragung auf andere Gotheiten selten ist, auffälligerweise (vgl. im folgenden die Situation bei $\alpha \dot{v} \alpha \varkappa \omega \varrho$ ) zuerst in der Tragödie

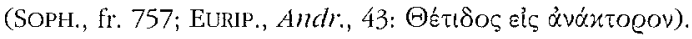


danach ist sie, im Plural, die Bezeichnung für den Palast. In det modernen Forschung wurde $\alpha \dot{\alpha} \alpha \dot{x} \tau$ togov, offenbar wegen der Existenz von $\alpha \dot{\alpha} \alpha \xi \xi$ (Stamm:

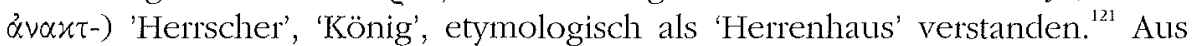
sprachwissenschaftlicher Sicht ist diese Deutung aber zurückzuweisen, da der Wortauslaut -oeov eine im Griechischen nicht existente Suffigietung enthalten müsste. ${ }^{122}$ Auch die Ansicht, ¿̇v'́xxtogov sei auf das im Mykenischen bezeugte Adjektiv wa-na-ka-te-ro (wanakteros 'königlich') zurückzuführen, ${ }^{123}$ ist nicht befriedigend, denn die dann anzunehmende lautliche Verschiebung von $-\tau \varepsilon \varrho O-z u-\tau O \varrho O-$ ist problematisch. Es ist freilich auch hier sehr viel einfacher, eine Interpretation zu entkräften, als dem Leser eine stichhaltige Lösung zu bieten. In der Tat ist das Wort àváx dig. Neben ảvóxutogov gibt es selten, zuerst bei den Tragikern, ${ }^{124}$ das nomen

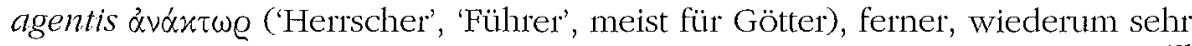

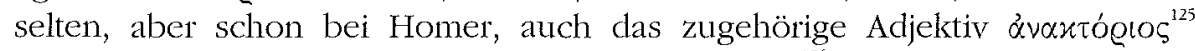
sowie später das Substantiv àvaxto@in 'Herrschaft'. ${ }^{126}$ Wie auch immer diese Wortfamilie etymologisch zu deuten ist, sie wirkt ausgesprochen altertümlich ${ }^{127}$ und scheint primär in den Kult zu gehören. Nach dem Ausscheiden der

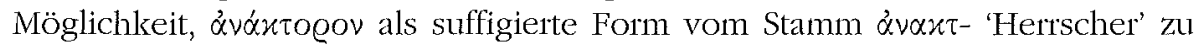
verstehen, bleibt, wenn wir auf den doch sehr naheliegenden Zusammenhang mit $\alpha^{\prime} \vee \alpha \xi$ nicht verzichten wollen, nur noch die Variante, in $\alpha \dot{v} \alpha \dot{x} \tau$ oeov ein altes Kompositum zu erblicken, das als solches im 1. Jt. nicht mehr durchsichtig war. Soweit ich sehe, gibt es dann aber nur eine sinnvolle Lösung, nämlich die Annahme einer zugrundeliegenden Form *wanakt-ktoron, die im Vorderglied den reinen Stamm des alten Wortes äva $\xi$ (myk. /wanaks/ 'König') ${ }^{128}$ und im Hinterglied denjenigen Stamm enthielte, der in $x \tau$ tég $\alpha_{\varsigma}$ bzw.

121 So L. Dfubner (1932), S. 87-90, der daraus den Schluss zieht, dass die Mysterien wohl einst im mykenischen Palast, und clann auch noch nach dem Zerfall der Königsherrschaft begangen worden seien. Auch LSJ, S. 110, s.v. גuáuto@ov, geht offenbar von der Deutung 'Herrenhaus' aus, da dort als Grundbedeutung „king's dwelling, palace“ angegeben wird.

${ }^{122}$ Im Dorischen gibt es auch eine Variante des Stammes ohne -t-: fóvaxes (für die Dioskuren, z.B. E. SCHWyzer [1923], nr. 79), favóxєı (Tempel der Dioskuren, z.B. E. ScHWyzFr [1923], nr. 350) u.a. Es ist wahrscheinlich, dass diese Variante sekundär ist, vgl. P. ChunTraine (1968ff.),

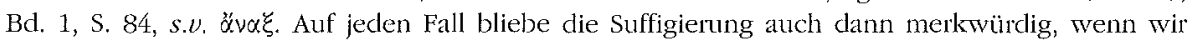
von ờúx-to@ov ausgingen.

123 C.J. RUIJGH (1967), S. 381f.

124 AIschrL., Choeph., 357; Eutrip., Iph. Tawn, 1414.

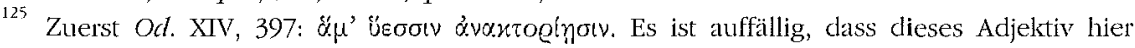
ausgerechnet für Schweine gebraucht wird, sind diese doch eng mit dem Demeterkult verbunden. In diesem Ausdruck eine ursprüngliche Bedeutung 'Scheine für den Kult im a.' erkennen zu wollen, bleibt aber eine weiter kaum stützbare Spekulation.

126 H.Ap. 234 (von Pferden); APOLL. RHOD., I, 839.

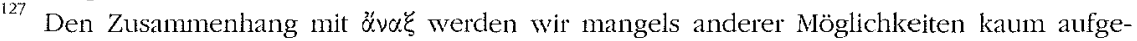
ben; $\not \alpha v \alpha \xi \xi$ ist aber im 1 . It. nur noch ein in der Sprache nicht melur lebendiges Relikt, s.u.

${ }^{126}$ Es könnte sich auch um die mit dem Kompositionsvokal -o erweiterte Form *wanaktohandeln, die durch Haplologie zu *wana-vereinfacht worden wäre. Die Ausbreitung von ${ }^{0}-$ als Kompositionsvokal auch ausserhalb der Komposita mit -o-Stämmen im Vorderglied scheint aber, nach dem mykenischen Material zu schliessen, eine nachmykenische Tendenz zu sein, die immerhin bei Homer schon gut entwickelt ist, vgl. E. Rusch (1974), S. $217 f$. 


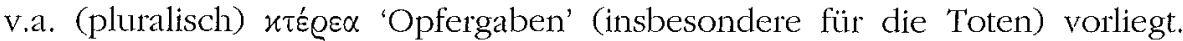
Auch an das seltsame Epitheton $\Delta \alpha_{\alpha} x \tau$ ogos lässt sich denken, das im Epos, oft in der Verbindung $\Delta \dot{\alpha} x \tau$ o Bedeutungsmässig wäre *wanaktoron angesichts einer für die Wurzel *kteranzunehmenden Kernbedeutung 'heiligen' o.ä. das 'Heiligtum des wanax', und $\alpha \dot{\alpha} \alpha \dot{\alpha} x \tau \omega \varrho$ mit seinem zweifellos sehr feierlich klingenden, altertümlichen Suffix $-\tau \omega \varrho^{130}$ müsste eine sekundäre Bildung der Tragiker sein. ${ }^{131}$

Nun war aber die Bezeichnung für den mykenischen König, wanax, nach dem Untergang der Paläste zusammen mit der Institution des Königtums dem Untergang geweiht. Obwohl wanax (in der Form $\alpha^{\prime} v \alpha \xi$ ) im 1. Jt. als Relikt aus dem 2. Jt. noch vereinzelt auftaucht und insbesondere im Epos eine gewisse Verbreitung findet, ist davon auszugehen, dass das Wort *wanakt(o?)-ktoron bzw. *wanaktoron aus der Zeit vor 1200 stammt, da nicht einsichtig ist, wie es nach 1200 hätte entstehen können. Die These, wonach die (weltlichen) Herrscher der mykenischen Königreiche auch als Götter verehrt wurden, ${ }^{132}$ scheint eine Stütze zu bekommen, da man kaum annehmen wird, der wanax sei der Eigentümer des wanaktorons, ohne darin verehrt zu werden. Dabei ist angesichts der Verbreitung des Titels wanax in der mykenischen Welt und deren kulturellen Einheitlichkeit vermutlich davon auszugehen, dass jeder mykenische Palast mit einem ávóx

Wie aber wird das 'Heiligtum des wanax' zum heiligsten Bereich im eleusinischen Demeterkult? Der bronzezeitliche Begriff wanaktoron mag nach 1200 als Name für ein ehemals besonders wichtiges Heiligtum in Erinnerung geblieben und später für das Allerheiligste in Eleusis verwendet worden sein. Die Wahl eines derart merkwürdigen Begriffs für eine im 1. Jt. neu entstehende Kultlokalität ist jedoch nicht sehr plausibel, weil $\dot{\alpha} \nu \alpha \dot{x} \tau o \varrho o v$ für die Sprecher der Dark Ages durchaus an das aus dem hexametrischen Epos (bzw. aus der Hymnendichtung) bekannte Wort $\alpha^{\prime} v \alpha \xi$ anklang, während mit dem $\alpha \dot{v} \alpha \dot{x}$ togov der historischen Zeiten offensichtlich kein $\alpha 2 v \alpha \xi$ in Verbindung stand.

Wahrscheinlicher ist es, mit einer gewissen Kultkontinuität zu rechnen, die evidenterweise nicht ungebrochen gewesen sein kann. Damit ist freilich die Frage nicht geklärt, ob sich das historische áváxtogov an derselben Stelle befand wie sein bronzezeitlicher Vorläufer. Es ist denkbar, dass sich im Rahmen der Wirren und Wanderbewegungen nach 1200 Flüchtlinge in Eleusis niederliessen, dort ihre früheren Kulte (teilweise) weiterführten und die alte Bezeichnung wanaktoron auf ein neues Gebäude übertrugen. Es ist aber ebensogut möglich, dass das historische áváxนo@ov direkt auf einen prähistorischen Vorläufer zurückzuführen ist. Die Argumente der Befürworter dieser

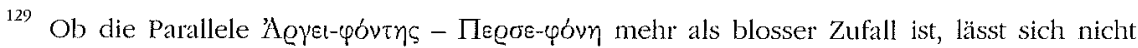
entscheiden.

130 P. ChANTRAINE (1933), S. 321-329; E. SCHWyzER (1939), S. 530 f.

131 Archaisierender Stil ist in der Tragödie nichts aussergewöhnliches: E.g. A. MELLLET, Aperçu d'une bistoire de la langue grecque, Paris, 1975", S. 217-227.

132 Z.B. P. LÉVÊQUE (1975), S. 44 f. 
letzteren Hypothese waren zwar nicht stichhaltig; ${ }^{133}$ das heisst aber nicht, dass die Hypothese als solche falsch ist.

\section{Zusammenfassung}

Die Resultate der obigen Untersuchung lassen sich wie folgt zusammenfassen: Erstens hat die etymologische Untersuchung der Bezeichnung $\Theta \varepsilon \sigma \mu^{-}$

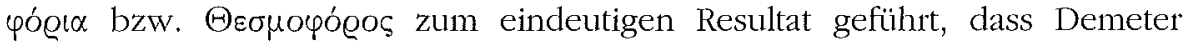

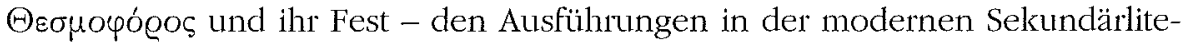
ratur zum Trotz - nichts mit Gesetzen zu tun haben kann. Denn die

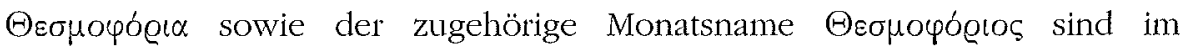
dorischen und böotischen Raum mit unzähligen Zeugnissen besonders gut belegt, ausnahmslos in der genannten lautlichen Form, im dorischen und

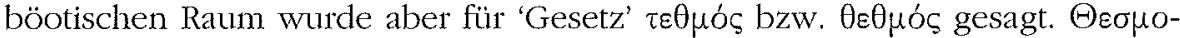
muss vielmehr auf eine alte Wurzel *dhes- 'heiligen' zurückgeführt werden und mit 'heiligen Gegenständen' zu deuten sein.

Zweitens wurde Demeter mit der mykenischen Göttin Potnia sowie mit Poseidon in Zusammenhang gebracht, Demeters bronzezeitliche Existenz unter den Namen $D \bar{a} \bar{o}$ bzw. Dāmāter hervorgehoben und die neu entdeckte mykenische Gottheit ma-ka aus Theben - entgegen der Meinung der Herausgeber des neuen mykenischen Textmaterials - von Demeter getrennt. Im bronzezeitlichen Theben kann keine Rede sein von einer Trias „Demeter Kore - Zeus".

Dafür hat allem Anschein nach die gut bekannte homerische Formel $\pi$ ótvı $\alpha$

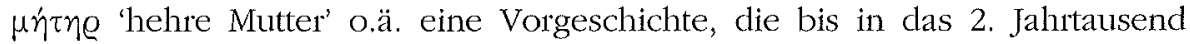
hinaufreicht. Diese Formel muss auf ein Syntagma zurückzuführen sein, in dem Potnia als Theonym und mêter als zugehörige Apposition zu verstehen ist. Denn anders lässt sich die Verwendung des Wortes $\pi$ ó $\tau v \iota \alpha$, das ursprünglich ein Substantiv war und im 2. Jt. der Name einer Gottheit gewesen sein muss, im homerischen Epos, wo es als adjektivisch gebrauchtes Beiwort für verschiedene göttliche Frauengestalten auftritt, kaum überzeugend erklären.

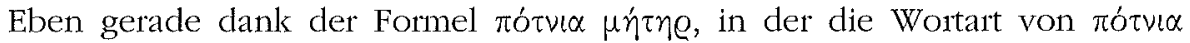
missverstanden werden konnte, muss das Theonym Potnia nach dem Verschwinden der so benannten Göttin zu einem Quasi-Adjektiv geworden sein ('Mutter Potnia' > 'hehre Mutter'). Das aber heisst, dass die epische

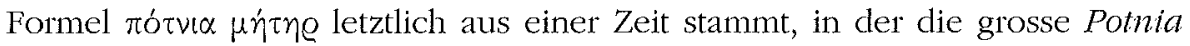
der Bronzezeit noch nicht in Vergessenheit geraten war. Dass dieses vielleicht ursprünglich aus der Hymnendichtung stammende Syntagma, wenn auch mit veränderter Bedeutung, den Weg ins Epos gefunden hat, unterstreicht die Wichtigkeit des Kultes der Demeter-Potnia.

Drittens glaube ich, in der anlässlich der eleusinischen Mysterien alljährlich vorgetragenen Verkündigung des Hierophanten einen Teil eines sehr alten

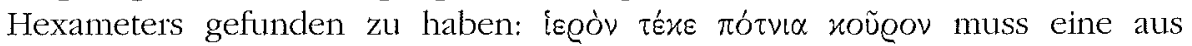

133 Dies zeigt deutlich P. DARCQUE (1981). 
prähistorischen Zeiten ererbte poetische Formel darstellen, die am ehesten einer aus den Dark Ages, vielleicht sogar aus der mykenischen Zeit ererbten Hymnentradition zuzuordnen ist. Auch gewisse Elemente des homerischen Demeterhymnus, v.a. die Namensform $\Delta \eta \omega \dot{\omega}$ des Theonyms und die Verwendung von $\ddot{v} v \alpha \sigma \sigma \alpha$ in einer hochtraditionell anmutenden hymnischen Formel (Vers 492) dü̈rften derselben Dichtungstradition angehören.

Viertens schlage ich vor, das sogen. ¿́váxтo@ov des eleusinischen Demeterkultes etymologisch als *(w)anakt-ktoron 'Heiligtum des (w)anax' zu deuten und damit seine Entstehung in mykenische Zeiten zu datieren. Das würde heissen, dass einerseits der mykenische wanax tatsächlich auch als Gottheit verehrt wurde, und dass andererseits der Demeterkult damit in Verbindung stand. Das Bindeglied scheint am ehesten in der Palastgöttin Potnia zu suchen zu sein, mit der der wanax zweifelsohne enge Kontakte pflegte. Demeter dürfte in einem alten wanaktoron das Erbe der Potnia angetreten haben, sei es in Eleusis, sei es anderswo: Neuzuzüger mögen nach 1200 ihren von anderswoher mitgebrachten Kult in Eleusis neu eingerichtet haben, oder aber es gab an Ort und Stelle einen bronzezeitlichen Vorgängerkult der eleusinischen Mysterien, der aber schon angesichts der damaligen Präsenz des mykenischen wanax mit Sicherheit mit dem späteren Kultgeschehen nicht identisch gewesen sein kann.

Wegen dieser Indizien, die den eleusinischen Demeterkult mit der später verschwundenen mykenischen Palastkultur in Zusammenhang bringen, ist es schliesslich wahrscheinlich, dass die eleusinischen Mysterien bedeutenderen Umformungen ausgesetzt waren als das Thesmophorienfest, obwohl letzteres, das an so zahlreichen Orten gefeiert wurde, eigentlich für Veränderungen eher hätte prädestiniert sein können. In beiden Fällen müssen wir aber damit rechnen, dass die Konstanz nicht in der jeweils unveränderten Weiterführung von Ritualen und Vorstellungen, sondern, genau wie im Falle einer Sprache, in deren kontinuierlichen Veränderungen liegt. Die vorliegende Untersuchung hat ein paar greifbare neue Indizien für die Vorgeschichte der Demeterkulte zu Tage gebracht, die, wie auch die griechische Sprache, im 2. Jt.v.C. in älteren Formen existiert haben mïssen.

Catherine TRÜmPY

Universität Heidelberg

Seminar für Klassische Philologie

Marstallhof, 2-4

D - 69117 HEIDELBERG 


\section{Abgekürzt zitierte Iiteratur:}

V.L. Aravantinos, L. Godart, A. Sacconi, Tbèbes, Fouilles de la Gadmée I, Les tableltes en linéaire $B$ de la Odos Pelopidou, Édition et commentaire, Pisa/Rom, 2001

J. BREMMER, Greek Religion, Oxford, 1994

A.C. Brumfield, The Attic Festivals of Demeter and their Relation to the Agricultural Year; Salem, New Hampshire, 1981

Ph. Bruneau, Recbercbes sur les cultes de Délos à l'époque bellénistique et à l'époque impériale, Paris, 1970

W. BUtkнkr, Homo necans, Inlerprelationen allgriechischer Op/erriten und Mythen, Berlin, 1972 [Neuauflage 1997]

W. BURKERT, Griechiscbe Religion der archaischen und klassiscben Epoche, Stuttgart, 1977

-, Greek Religion, Cambridge, Massachusetts, 1985, engl. Übersetzung von W. Burkekr (1977)

-, Ancienl Mystery Calls, Cambridge, Massachusetts/London, 1987

P. Chantraine, La formation des noms en grec ancien, Paris, 1933

-, Dictionnaire étymologique de la langue grecque, 2 Bd., Paris, $1968 f f$.

K. Clinton, „The Thesmophorion in Central Athens and the Celebration of the Thesmophoria in Attica“, in R. HÄGG (ed.), The Role of Religion in the Earty Greek Polis, Stockholm, 1996

P. DARCQUe, „Les vestiges mycéniens découverts sous le téléstérion d'Éleusis“, BCH 105 (1981), S. 595-605

N. DFMand, Birth, Death, and Motherbood in Classical Greece, Baltimore, 1994

M. Detienne, „Violentes 'eugénies'. En pleines Thesmophories: des femmes couvertes de sang“, in M. Detienne - J.-P. Vernant (eds.), La cuisine du sacrifice en pays grec, Paris, 1979, s. 183214

-, "The Violence of Wellborn Ladies: Women in the Thesmophoria", in M. DeTIEnNe, J.-P. VERNANT (eds), The Cuisine of Sacrifice among the Greeks, Chicago/London, 1989, S. 129147, engl. Übersetzung von M. DETIENNE (1979)

L. DEuBNER, Attische Feste, Berlin, 1932 (Neuauflagen 1966 und 1969)

B.C. DIETRICH, Origins of Greek Religion, Berlin, 1974

-, Tradition in Greek Religion, Berlin, 1986

DNP: Der Neue Pauly 1-12, eds H. CANCIK und H. SCHNEIDER, Stutgart/Weimar; 1996-2003

V. EHRINBERG, Die Recbtsidee im, /rïhen Griechentum, Leipzig, 1921

L.R. FARNELL, The Cults of the Greek States III, Oxford, 1906

H.P. FOLEY, The Homeric Hymn to Demeter; Princeton, 1994

H. FusK, Griechisches Etymologisches Wönerbuch, 2 Bd., Heidelberg, 1960/70

M. GERARD-ROUSSEAU, Les mentions religieuses dans les tablettes mycéniennes, Rom, 1968

L. GODART, A. SACCONI, "Tebe, Demetra ed Eleusi“, in P.A. BERnardini (ed.), Presenza e Funzione della Città di Tebe nella Cultura Greca, Alli del Convegno Internazionale (7-9.Juglio 1997), Rom, 2000, S. 17-26

F. GRAF, Eleusis und die orpbische Dichtung Atbens in vorbellenistischer Zeit, Berlin, 1974

F. Gschnitzer, „Zum Namen Poseidon“, in R. MUTH in Verbindung mit F. GSCHNITZER, F. HANPL, J. KNOBLOCH (eds), Serla philologica Aenipontana, Innsbrucker Beiträge zur Kultumissenschaft 7-8, Innsbruck, 1962, S. 13-18

-, „Zur Terminologie von 'Gesetz' und 'Recht' im frühen Griechisch", in G. THür et al. (eds), Symposion 1995, Vortrigge zur griechischen und bellenistiscben Recbtsgescbicbte, Köln, 1997 , S. 3-10

-, Kleine Scbrịten zum griecbischen und römischen Altertum, Band I. Frïbes Griechentum: Historiscbe und spracbwissenscbafticbe Beiträge (eds C. TRÜNPY - T. SCHMTT), Stuttgart 2001

M. JANDA, Eleusis, Das indogermanische Evbe der Wysterien, Innsbruck, 2000

M. Jost, Sanctuaires et cultes d'Arcadie, Paris, 1985 
M. LEJSunE, Phonétique bistorique du mycénien et du grec ancien, Paris, 1972

A. LESKY, Geschichte der griechischen Literatur; Bern, $1971^{3}$

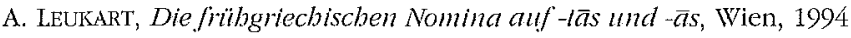

P. LÉVÊQUE, „Le syncrétisme créto-mycénien“, in F. DUNAND - P. LÊVÊQUE (eds), Les syncrétismes dans les religions de l'antiquité, colloque de Besançon (22-23 octobre), Leiden, 1975

LSJ: H.G. LIDDELL, R. SCOTT, H.S. JONES, A Greek-English Lexicon, Oxford, 1968

M. MAYRHOFER, Kurzgefasstes etymologisches Wörterbuch des Altindischen, Bd. 2, Heidelberg, 1963 —, Indogermanische Grammatik, 2. Halbband: Lautlehre, Heidelberg, 1986

M.P. NiLSSON, Griechische Feste von religiöser Bedeutung mit Ausschluss der attischen, Leipzig, 1906

—, Gescbicbte der griecbiscben Religion $\mathrm{I}^{3}$, München, 1967

H.W. Parke, Festivals of the Athenians, London, 1977

J.-L. PERPILlou, Les substantifs grecs en -aus, Paris, 1973

H. PFTERSMANN, „Altgriechischer Mütterkult", in Matronen und verwandte Gottbeiten. Ergebnisse eines Kolloquitums, veranstaltet von der Göttinger Akademiekommission fill die Altertumskunde Mittel- und Nordeuropas, Köln, 1987 (BJ, Beiheft 44), S. 171-199.

-, Lingua et Religio, Ausgewählte Kleine Schriften zur anliken Religionsgescbicbte auf sprachwissenschafticher Grundlage (ed. B. HESSEN), Göttingen, 2002

PLG III': Th. Bergk, Poetae Lyrici Graeci $\mathrm{III}^{1}$, Leipzig, 1882

PMG: Poetae melici Graeci, ed. D.L. PAGE, Oxford, 1962

N.J. RICHARDSON, The Homeric Hymn to Demeter; Oxford, 1974

E. RISCH, Wortbildung der bomeriscben Spracbe, Berlin, $1974^{2}$

-, Kleine Schriflen zum siebzigsten Geburtstag, eds A. ETTER - M. LOOSER, Berlin, 1981

C.J. RuJGH, Etudes sur la grammaire et le vocabulaire du grec mycénien, Amsterdam, 1967

E. SCHWYZER, Dialectonum Graecarum exempla epigraphica potiora, Leipzig, 1923

-, Griechische Grammatil I, München, 1939

-, Griecbiscbe Grammatik II, München, 1950

A.L. StHLER, New Comparative Grammar of Greek and Latin, New York/Oxford, 1995

E. SImon, Festivals of Attica, An Archaeological Commentary, Madison, Wisconsin, 1983

-, Die Gölter der Griechen, Würzburg, $1985^{3}$

C. TRÜMPY, Untersuchungen zu den altgriechischen Monatsnamen und Monatsfolgen, Heidelberg, 1997

- "Potnia dans les tablettes mycéniennes: quelques problèmes d'interprétation", in R. LAFFINEUR - R. HÄGG (eds), Potnia, Deities and Religion in the Aegean Bronze Age, Proceedings of the 8th International Aegean Conference Göteborg, Göleborg University, 12-15 April 2000, Liège, 2001 (Aegaeum, 22), S. 411-421

H.S. VERSNEL, Inconsistencies in Greek and Roman Religion II, Transition and Reversal in Mytb and Ritual, Leiden, 19932, S. 235-288

J.J. WINKLER, The Constraints of Desire: The Antbropology of Sex and Gender in Ancient Greece, New York, 1990, S. 193-202

F.I. ZEITLIN, „Travesties of Gender and Genre in Aristophanes' Thesmophoriazousai“, in H.P. FolEY (ed.), Reflections of Women in Antiquity, New York et al., 1981, S. 169-217

-, "Cultic models for the Female: Rites of Dionysus and Demeter", Arethusa 15 (1982), S. 129157. 


\section{Résumé. Les Thesmophories, Brimo, Deo et l'anaktoron : quelques observations sur la préhistoire du culte de Déméter}

Déméter et sa fille, qui étaient vénérées dans toutes les régions de la Grèce, comptent sans doute parmi les divinités les plus populaires que l'on puisse trouver au $\mathrm{I}^{\mathrm{er}}$ millénaire av. J.-C. S'il s'agit d'éclaircir la préhistoire de leur culte, ce sont les Thesmophories et les mystères d'Éleusis qu'il faut étudier en premier lieu dans la mesure où, dans ces deux cas, nous n'avons pas seulement une documentation assez riche, mais où nous entrevoyons également certaines caractéristiques qui témoignent de leur origine lointaine.

Étant attestées dans la quasi-totalité des cités de même que dans les parties les plus isolées du monde grec, les Thesmophories sont la fête la plus répandue. Ainsi, il est impensable qu'elles aient été établies à l'époque historique. Au contraire, il doit s'agir d'un bien culturel hérité d'une époque antérieure à la séparation des Grecs en groupes ethniques différents, ce qui nous mène au $\mathrm{II}^{2}$ millénaire. L'étymologie du nom des Thesmophories que je propose confirme cet âge reculé. Il est vrai que - $\varphi$ Q- contient la racine bien connue signifiant 'porter' (*pher-), mais le sens de $\theta \varepsilon \sigma \mu o-$ est beaucoup

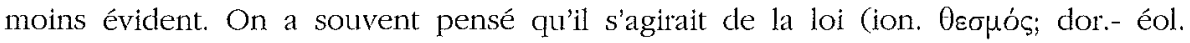

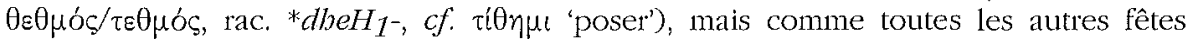
contenant -phoria dans leur nom s'appliquent à des objets concrets, cette interprétation est a priori peu probable, d'autant plus que dans nos sources relatives aux cultes, Déméter n'a tien à voir avec la législation. Ceux qui supposent que les $\theta \varepsilon \sigma \mu o-s o n t$ des 'objets posés' n'obtiennent pas un sens plus satisfaisant non plus parce que durant les Thesmophories, personne ne porte quoi que ce soit de 'posé'. De toutes façons, il existe un argument d'ordre dialectologique qui prouve que thesmo- doit être séparé de $\tau i \theta \eta \mu$ : en effet, les Thesmophories, dont le nom est attesté à d'innombrables reprises, notamment dans celles des régions où les lois apparaissent sous la forme de $\theta \varepsilon \theta \mu o i ́ /$

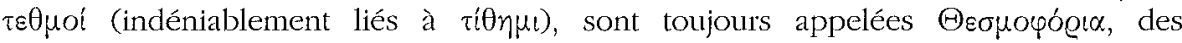

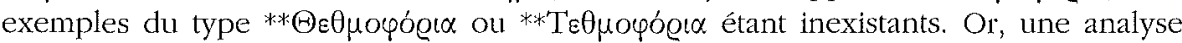
systématique du matériel comparable $(\theta \varepsilon \delta \varsigma$, , $\theta \dot{\varepsilon} \sigma \varphi \alpha \tau o \varsigma$, lat. fèriae, festus, fānum, sanscr: dhis-) m'a amené à postuler une vieille racine *dhes- 'rendre sacré', saisissable dans plusieurs langues indoeuropéennes, mais obscurcie en grec classique. Étymologiquement parlant, les Thesmophories étaient donc la fête durant laquelle certaines personnes devaient porter des objets sacrés, ce qui correspond bien à ce que nous savons par ailleurs à propos des rites accomplis lors de leur déroulement.

Du point de vue linguistique, Déméter (forme ancienne : Dā-mātêr) et Poséidon (myc. $/$ Posei-dā-on $n$ ) doivent être considérés comme deux divinités étroitement liées. Au II millénaire, Déméter devait être vénérée sous le nom de $D \bar{a}$ ou $D \bar{a} \bar{o}$ alors que Poseidāōn était son époux. En outre, comme le montrent son étymologie ainsi que l'évidence des tablettes mycéniennes, ce dernier était aussi en rapport avec Potnia, déesse mycénienne plus ou moins identique à Déméter, mais visiblement en relation avec le palais royal, ce qui explique sa disparition après la chute des royaumes mycéniens. En ce qui concerne ma-ka, déesse mycénienne récemment découverte à Thèbes et interprétée également comme Déméter ( $M \bar{a} G \bar{a}$ 'Grande Déesse'), il convient de rejeter cette identification, notamment pour des raisons linguistiques. En effet, la dentale $D$ - de Déméter assurée aussi pour l'âge du Bronze est incompatible avec la

* $N d l r$ : la publication de cette étude ayant été initialement prévue en français, l'auteur a adjoint, à notre demande, ce résumé substantiel. 
gutturale $G$ - de $G a ̈$ 'terre'. Par ailleurs, Déméter est loin d'être une "Terre Mère ». En réalité, $m a-k a$ a de bonnes chances d'avoir été une vieille divinité locale tout à fait importante (Marga? Manga? Manka? etc., peut être $M \bar{a} G \bar{a}$ ), mais distinguée de Déméter qui, en tant que Potnia, apparaît également dans les tablettes de Thèbes. Quant à o-po-re-i qui est attesté à côté de $m a-k a$, les éditeurs des nouveaux textes thébains le considèrent comme le datif d'un Zeus Opōrēs " protecteur des fruits de la terre », et ko-wa/korwa $/$, qui, tout en étant suivie d’autres destinataires d'offrandes, est mentionnée après ma-ka et o-po-re-i, est censée correspondre à Kógn; à leurs yeux, ma-ka, o-po-re-i et ko-wa correspondraient aux trois divinités de la fameuse triade d'Éleusis. Or, pour des raisons morphologiques, o-po-re-i doit être séparé du terme ó $\tilde{\omega} \varrho \alpha$ 'automne' puisque ce dernier est uniquement attesté comme thème en $-\bar{a}$ qui ne saurait être à la base d'un Opōrēs qui contient un thème en -s. De nouveau, il s'agit probablement d'une vieille divinité locale sans étymologie, ce qui a pour conséquence qu'il n'y a plus aucune raison de considérer ko-ua comme le nom propre de la fille de Déméter.

Potnia se retrouve dans la formule homérique $\pi$ ótvi $\alpha$ ṙंne qui doit constituer un des éléments les plus anciens de la langue homérique. En effet, pour plusieurs raisons linguistiques, cette formule doit remonter à un syntagme Potnia (théonyme) mātêr (apposition) - syntagme exactement parallèle à celui de $D a \bar{a}-m \bar{a} t \bar{e}$. (Déméter). Or, la déesse Potnia n'a pas survécu à la chute des palais mycéniens ce qui veut dire que la

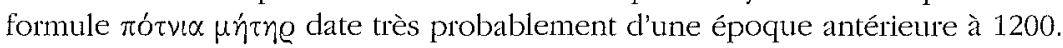

Lors des mystères d'Éleusis, l'hiérophante devait annoncer la naissance d'un enfant

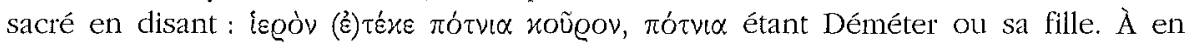
juger non seulement par la présence de Potnia et d'une métrique poétique, mais également par l'hyperbate (iॄgòv ... xoũgov) et la forme homérique, non éleusinienne, de roṽ oov, il doit s'agir d'une partie d'hexamètre hautement archaïque appartenant probablement à une vieille tradition hymnique saisissable aussi dans l'hymne homérique dédié à Déméter.

Finalement, je propose de voir dans le fameux ávóxto@ov, bâtiment le plus sacré d'Éleusis, un *(w)anakt-ktoron 'sanctuaire du (w)anax' (*kter-/*ktor- comme dans

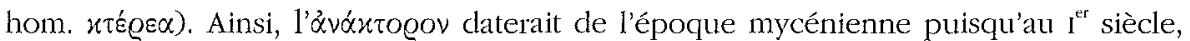
le mot $\alpha \dot{\alpha} \alpha \xi \xi$ n'est plus qu'une réminiscence tombée en désuétude dans la langue parlée. Si nous acceptons cette analyse étymologique, il est raisonnable d'admettre que le wanax mycénien était honoré non seulement en tant que souverain politique, mais également comme dieu qui semble avoir été lié à Potnia (-Déméter), ce qui explique

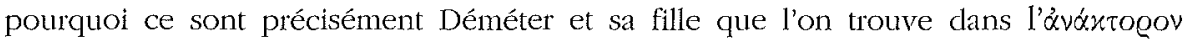
d'Éleusis. Celles-ci ont dû prendre la succession de Potnia dans un ảvóxto@ov préexistant à Éleusis ou ailleurs et s'installer définitivement à Éleusis. 